U.S. DEPARTMENT OF THE INTERIOR

\author{
U.S. GEOLOGICAL SURVEY
}

\title{
THE CONTRIBUTION OF GIANT FIELDS TO UNITED STATES OIL PRODUCTION AND RESERVES
}

by

James W. Schmoker

Open-File Report 99-131.

This report is preliminary and has not been reviewed for conformity with U.S. Geological Survey editorial standards or with the North American Stratigraphic Code. Any use of trade, product or firm names is for descriptive purposes only and does not imply endorsement by the U.S. Government.

U.S. Geological Survey, Mail Stop 939, Denver Federal Center, Denver, CO 80225 


\section{INTRODUCTION}

In the U.S., an oil field is regarded as "giant" if the sum of cumulative production and remaining (proved) reserves exceeds 100 million barrels of oil (mmbo). Giant fields account for a very small fraction of the total number of oil fields discovered in the U.S., but their importance to U.S. production - past and future - vastly exceeds their numerical proportion.

This report presents and interprets production and reserves data for giant U.S. oil fields, for the purpose of better understanding and forecasting U.S. oil production and the U.S. resource base. The primary data used here are arranged by location in two tables. Table 1 provides data for giant U.S. oil fields as of 1/1/98: this table contains field name and location; year of discovery; annual production in 1997; cumulative production, remaining reserves, and the sum of these two quantities (estimated total recovery) as of 1/1/98; and the ratio of remaining reserves to 1997 annual production. Table 2 lists those fields of Table 1 for which production and reserves data were also published in 1990: this table introduces columns for cumulative production, remaining reserves, and estimated total recovery as of $1 / 1 / 90$; and the change in estimated total recovery between $1 / 1 / 90$ and $1 / 1 / 98$.

The data of Table 1 offer a snapshot of production and reserves characteristics of giant fields at one point in time. The estimated size (cumulative production plus proved reserves) of an oil field can change significantly over a relatively short time span because proved reserves are dependent upon factors that do not remain constant: the data of Table 2 capture changes in the sizes of many of these giant fields in the first eight years of the 1990's.

\section{PRODUCTION AND RESERVES AS OF 1/1/98}

The list of giant U.S. oil fields presented in Table 1, derived from the International Petroleum Encyclopedia (1998), is probably not exhaustive but appears to be reasonably complete. The implicit assumption is made here that Table 1 is sufficiently inclusive to warrant analysis. Table 1 could be incomplete due to: 1) the general vagaries of data collection and data bases; 2) omission of older fields that recently attained giant-field status; and 3) omission of recently discovered large fields.

\section{Perspectives}

The 293 giant U.S. oil fields, of which 16 have estimated total recoveries exceeding 1 billion barrels of oil (bbo), account for about $58 \%$ of the discovered oil in the U.S., and for $59 \%$ of cumulative U.S. oil production (Figure 1). These fields have contributed substantially to the economic development of the U.S.

As of $1 / 1 / 98$, the estimated total recovery of oil from all giant fields in the U.S. was 113.1 bbo (Figure 1), which is roughly equivalent to that of Iran or Kuwait (see Masters and others, 1994). Only the Former Soviet Union has produced more oil (128.1 bbo) than the 101.8 bbo produced from giant U.S. oil fields (International Petroleum Encyclopedia, 1998). The giant U.S. oil fields, of themselves, would constitute a world-class oil "country".

The median discovery year for giant U.S. oil fields is 1935 , and $90 \%$ of their recoverable oil has been produced (Figure 1). Their median and average ratios of remaining oil reserves to annual oil production are low. These data show that the giant oil fields of the U.S. form a mature 
producing sector. However, production from these fields is still significant, even on a world scale. Production from giant U.S. oil fields averaged $3.65 \mathrm{mmbo}$ /day in 1997, which is equal to $57 \%$ of daily U.S. oil production (Figure 1) and is a rate exceeded worldwide only by Saudi Arabia and the Former Soviet Union (International Petroleum Encyclopedia, 1998).

\section{Fields Having Significant Remaining Potential}

The previous discussion did not differentiate between giant fields which have significant remaining potential and those which appear to be in terminal decline. The former fields represent the future of the group; the latter fields have become, at a National perspective, part of history.

The remaining potential of giant U.S. oil fields is evaluated here on the basis of remaining reserves (as of 1/1/98) and annual production (in 1997). Figure 2 shows that, for both measures of remaining potential, relatively few fields account for most of the oil.

Fifty percent of 1997 annual production from all giant U.S. oil fields came from just 11 fields and $80 \%$ was supplied by 55 fields (Figure 2A). The distribution of remaining reserves is yet more uneven. As of $1 / 1 / 98,50 \%$ of remaining reserves in all giant U.S. oil fields resided in just six fields and $80 \%$ was accounted for by 42 fields (Figure $2 \mathrm{~B}$ ).

Using the somewhat arbitrary criteria that giant fields having significant remaining potential are those that account for either $80 \%$ of total annual production or $80 \%$ of total remaining reserves, 59 giant U.S. oil fields are identified here as having significant remaining potential (Table 3). Of these, 38 fields qualify with respect to both annual production and remaining reserves.

The giant U.S. oil fields having significant remaining potential (Table 3) form a diverse group. They are distributed among 10 states, although most are located in Texas, California, and Louisiana (Figure 3). Common geologic features among these fields, other than access to an excellent oil-charge system, are not obvious, nor are they obvious for giant U.S. oil fields in general.

\section{Field-Size and Oil-Volume Distributions}

The field-size distribution of the 293 giant U.S. oil fields listed in Table 1 is extremely skewed (Figure 4A). The coefficient of skewness is 9.1. (A skewness coefficient greater than 1.0 is commonly considered to indicate a highly skewed distribution.) Note that the eye tends to be fooled by Figure 4A: the logarithmic size-category axis, necessary to construct a legible figure, greatly foreshortens the larger field-size categories and thus reduces the apparent skewness.

One hundred and fifty-one fields (almost 52\%) have sizes between 100 and $200 \mathrm{mmbo}$, whereas only eight fields (about $3 \%$ ) have sizes in the much larger range between 1,600 and $12,800 \mathrm{mmbo}$ (Figure 4A). The biggest field of the group (Prudhoe Bay) is about 65 times larger than the median field of $194 \mathrm{mmbo}$. If this ratio were to be applied to the height of humans, a median adult height of 5.5 feet would imply the existence of people as tall as 358 feet.

Approximately equal volumes of oil are attributed to field-size categories 1, 2, and 3 (Figure 4B). The volume of oil ascribed to field-size categories 4 through 7 tends to decrease as 
size category increases. (Data for size categories 6 and 7 are erratic because each category contains only one field.)

The U.S. is the most thoroughly explored large petroleum region in the world. As such, the field-size and oil-volume distributions for discovered giant U.S. oil fields (Figure 4) might provide as close an approximation to a natural (in situ) population of such fields as is presently known. For this reason, the distributions of Figure 4 could have value as analogs for some of the less explored regions of the world.

\section{CHANGES IN FIELD SIZES BETWEEN 1/1/90 AND 1/1/98}

The sizes (estimated total recoveries) of U.S. oil fields commonly change over time, in response to a large number of geological, technical, economic, and regulatory factors (e.g., Arrington, 1960; Attanasi and Root, 1994; Gautier and others, 1995; Schmoker and Crovelli, 1998). Recent changes in the sizes of 209 giant U.S. oil fields (of the 293 fields considered in the previous sections) can be examined here, using the production and reserves data as of 1/1/90 that are provided in Table 2.

\section{Field-Size Changes Measured as Percentages}

The great majority (96\%) of the fields listed in Table 2 were 20 or more years old at the beginning of 1990; about two-thirds were discovered prior to 1941 (Figure 5). Almost all the fields are well past the period of initial development in which large changes in estimated total recovery can be expected to occur as a field is delineated and production practices are optimized. All U.S. fields are subject to a standard system for reporting remaining reserves, which minimizes capricious field-size changes. One might therefore think that after many years of development and production, the remaining potential of most of these giant oil fields should be well established, but Figure 6 shows that this is not the case.

Of the 209 fields in the data set of Table 2, the size of only 30 fields (14\%) remained essentially unchanged on a percentage basis (larger or smaller by $0.5 \%$ or less) between $1 / 1 / 90$ and $1 / 1 / 98$ (Figure 6A). Sixty-five fields ( $31 \%$ ) increased or decreased in size by 0.5 to $4 \%$ in this eight-year period, 52 fields $25 \%$ ) changed in size by 4 to $10 \%$, and 62 fields (30\%) changed in size by more than $10 \%$. (These percentage changes are relative to $1 / 1 / 90$ sizes.) From these data it is apparent that significant adjustments in estimated total recovery have been the norm, and not the exception, for giant U.S. oil fields in the first eight years of the 1990's.

Furthermore, Figure 6A shows that field-size increases outnumbered field-size decreases by more than two to one. Excluding the 30 fields with little percentage change, 126 fields increased in size whereas 53 fields decreased in size.

Production and reserves data as of 1/1/90 are available for 48 of the 59 giant U.S. oil fields having significant remaining potential. The percent-change distribution for these 48 fields (Figure 6B) is weighted more heavily to the right than the distribution for the entire group of giant fields (Figure 6A). Of the fields with significant remaining potential, the size of seven fields remained essentially unchanged on a percentage basis, 37 fields increased in size, and only four fields decreased in size (Figure 6B). More than half of these fields (54\%) increased in size by over $10 \%$. 
The group of giant U.S. oil fields having significant remaining potential has been buoyed by large recent increases $(1 / 1 / 90-1 / 1 / 98)$ in field sizes (Figure 6B). These large field-size increases could be the result of high annual production rates; conversely, large field-size increases could drive annual production rates. Cause and effect are here complexly interwoven.

\section{Field-Size Changes Measured as Barrels of Oil}

Of the 209 giant U.S. oil fields in the data set of Table 2, the size of 29 fields (14\%) increased or decreased by only 1 mmbo or less between 1/1/90 and 1/1/98 (Figure 7A). Sixtyseven fields $(32 \%)$ increased or decreased in size by 1 to $10 \mathrm{mmbo}$ in this eight-year period, 73 fields (35\%) changed in size by 10 to $40 \mathrm{mmbo}$, and 40 fields $(19 \%)$ changed in size (larger or smaller) by more than $40 \mathrm{mmbo}$. Changes in estimated total recovery of tens of millions of barrels were common for giant U.S. oil fields in the first eight years of the 1990's (Figure 7A).

The distribution of Figure 7A is weighted toward the right, indicating that field-size increases have added substantially more oil than has been lost by field-size decreases. A number of giant U.S. oil fields have had extraordinarily long reserves lives and are declining at rates below expectations, largely because of the tendency for their estimated total recoveries to increase with time (Hatcher and Tussing, 1997).

The net change in estimated total recovery of the 209 giant U.S. oil fields contributing to Figure 7A (that is, the sum of the 209 individual field-size changes) is an increase of 5,896 mmbo. This volume is equal to 2.5 years of U.S. oil production, assuming average daily production at the 1997 rate of $6.40 \mathrm{mmbo}$ /day (Figure 1).

Of the 48 giant U.S. oil fields having significant remaining potential that are contributing to Figure 7B (a subset of the 59 fields of Table 3), 35 (73\%) increased in size by more than 20 mmbo in the first eight years of the 1990's. The net change in estimated total recovery of these 48 fields is an increase of $6,439 \mathrm{mmbo}$ (compared to a net increase of 5,896 mmbo for the parent set of 209 giant fields).

As shown in Figure 7B, the size of two fields having significant remaining potential increased by more than 1,000 mmbo between 1/1/90 and 1/1/98: these Alaskan fields are Prudhoe Bay (2,631 mmbo) and Kuparuk River (1,094 mmbo). The 12 fields of Figure 7B with field-size increases between 100 and 1,000 mmbo are Midv/ay-Sunset (440 mmbo, California), Wasson (288 mmbo, Texas), Belridge S. (268 mmbo, California), Spraberry Trend (201 mmbo, Texas), Slaughter (176 mmbo, Texas), Endicott (153 mmbo, Alaska), Seminole (148 mmbo, Texas), Levelland (130 mmbo, Texas), Rangely (117 mmbo, Colorado), Sho-Vel-Tum (115 mmbo, Oklahoma), Giddings (113 mmbo, Texas), and Cowden N. (111 mmbo, Texas). At the other extreme, East Texas field (Texas) decreased in size by $616 \mathrm{mmbo}$, and Elk Hills field (California) decreased by 65 mmbo.

\section{Field-Size Changes by Location}

Regional differences in patterns of field-size changes exist among the 209 giant U.S. oil fields of Table 2 (Figure 8). The giant fields of five areas had a net increase in estimated total recovery between $1 / 1 / 90$ and $1 / 1 / 98$, whereas the giant fields of four areas had a net decrease (Figure 8). The North Slope of Alaska provided the largest net increase, followed by the Permian Basin of West Texas and New Mexico, the San Joaquin Basin of southern California, 
and basins of the Midcontinent. The Los Angeles Basin and coastal areas of California (area 5) showed a positive but relatively insignificant net change in estimated total recovery.

Each of the three areas that encompass the U.S. Gulf Coast (areas 6, 7, and 9) had net decreases in estimated total recovery of giant fields (Figure 8). The net change in area 9 would be negative even if the East Texas field (which decreased in size by $616 \mathrm{mmbo}$ ) were excluded. In the Rocky Mountains (area 8), only three of 19 giant fields decreased in size, but these decreases were large enough to place the area into the negative-change category.

Producing areas within the U.S. differ from one another with respect to factors affecting field-size changes, such as geologic characteristics, oil-field practices, and perhaps reservesreporting patterns. Figure 8 illustrates how these differences can lead to very dissimilar regional perceptions of the volume of discovered but yet-to-be-produced oil in the U.S.

\section{SUMMARY}

1. In the U.S., a giant oil field is one whose size exceeds $100 \mathrm{mmbo}$. This study examines 293 giant fields (Table 1), using data as of 1/1/98. Recent changes in the sizes of 209 of these fields (Table 2) are also investigated, using production and reserves data as of 1/1/90.

2. Giant oil fields are still important to the U.S. energy picture (Figure 1). Average daily production in 1997 from giant fields was $3.65 \mathrm{mmbo} /$ day ( $57 \%$ of total U.S. oil production), and remaining reserves were 11.3 bbo ( $50 \%$ of total U.S. remaining oil reserves).

3. Among the 293 giant U.S. oil fields, 59 fields account for $80 \%$ of total annual production (in 1997 ) and $80 \%$ of total remaining reserves (as of 1/1/98). These 59 fields (Table 3 ) are identified here as giant fields having significant remaining potential.

4. The field-size distribution of giant U.S. oil fields (Figure 4), which provides a possible analog for some less explored regions of the world, is highly skewed. One hundred and fiftyone fields have sizes between 100 and $200 \mathrm{mmbo}$, whereas only eight fields have sizes in the much larger range between 1,600 and $12,800 \mathrm{mmbo}$.

5. Although the median discovery year of giant U.S. oil fields is 1935 , changes in field size (larger or smaller) of tens of millions of barrels were common between 1/1/90 and 1/1/98. The number of fields that increased in size outnumbered those that decreased in size by more than two to one (Figures 6,7).

6. Changes in individual field sizes in the first eight years of the 1990's were highly variable. Some of this variability is related to regional location (Figure 8). Overall, the estimated total recovery of the 209 giant U.S. oil fields for which necessary data are available for analysis increased by $5,896 \mathrm{mmbo}$, equal to about 2.5 years of current U.S. oil production. The estimated total recovery of a subset of 48 giant fields having significant remaining potential increased by $6,439 \mathrm{mmbo}$. 


\section{REFERENCES CITED}

Arrington, J.R., 1960, Predicting the size of crude reserves is key to evaluating exploration programs: Oil \& Gas Journal, v.58, no. 9 (February 29), p. 130-134.

Attanasi, E.D., and Root, D.H., 1994, The enigma of oil and gas field growth: American Association of Petroleum Geologists Bulletin, v. 78, no. 3, p. 321-332.

Gautier, D.L., Dolton, G.L., Takahashi, K.I., and Varnes, K.L., eds., 1995, 1995 National assessment of United States oil and gas resources - Results, methodology, and supporting data: U.S. Geological Survey Digital Data Series DDS-30.

Hatcher, D.B., and Tussing, A.R., 1997, Long reserves lives sustain prospects for independents in the U.S. lower 48: Oil \& Gas Journal, v. 95, no. 46 (November 17), p. 49-59.

International Petroleum Encyclopedia, 1990, U.S. fields with reserves exceeding 100 million bbl (data table): Tulsa, OK, PennWell Publishing Co., 378 p.

International Petroleum Encyclopedia, 1998, U.S. fields with ultimate oil recovery exceeding 100 million bbl (data table): Tulsa, OK, PennWell Publishing Co., [CD-ROM].

Masters, C.D., Attanasi, E.D., and Root, D.H., 1994, World petroleum assessment and analysis: Proceedings of the $14^{\text {th }}$ World Petroleum Congress, Chichester, England, John Wiley and Sons, v. 5, p. 529-541.

Schmoker, J.W., and Crovelli, R.A., 1998, A simplified spreadsheet program for estimating future growth of oil and gas reserves: Nonrenewable Resources, v. 7, no. 2, p. 149-155. 


\section{General}

- Number and estimated total oil recovery of all giant fields:

- Number and estimated total oil recovery of giant fields $>1$ bbo:

- Estimated total U.S. oil recovery:

- Median discovery year of giant fields:

- Median size of giant fields:

- Average size of giant fields:
293 fields, 113.1 bbo

16 fields, 42.4 bbo

195.6 bbo

1935

$194 \mathrm{mmbo}$

$386 \mathrm{mmbo}$

\section{Annual and Cumulative Production}

- Average daily oil production in 1997 from all giant fields:

$3.65 \mathrm{mmbo} /$ day

- Average daily U.S. oil production in 1997:

$6.40 \mathrm{mmbo} /$ day

- Average daily U.S. oil consumption in 1996:

$17.40 \mathrm{mmbo} /$ day

- Cumulative oil production from all giant fields:

101.8 bbo

- Cumulative U.S. oil production:

173.2 bbo

\section{Remaining Reserves}

- Remaining (proved) oil reserves in all giant fields:

11.3 bbo

- Remaining (prov: 1) r. . o oil reserves:

22.4 bbo

- Median remaining oil reserves/annual oil production ratio of giant fields:

5.7 years

- Average remaining oil reserves/annual oil production ratio of giant fields:

8.3 years

- Average remaining U.S. oil reserves/annual U.S. oil production ratio:

9.6 years

Figure 1. Numerative overview of giant U.S. oil fields (fields larger than $100 \mathrm{mmbo}$ ) as of 1/1/98. Data are from Table 1 and International Petroleum Encyclopedia (1998).

$[\mathrm{mmbo}=$ million barrels of oil. bbo $=$ billion barrels of oil. $]$ 


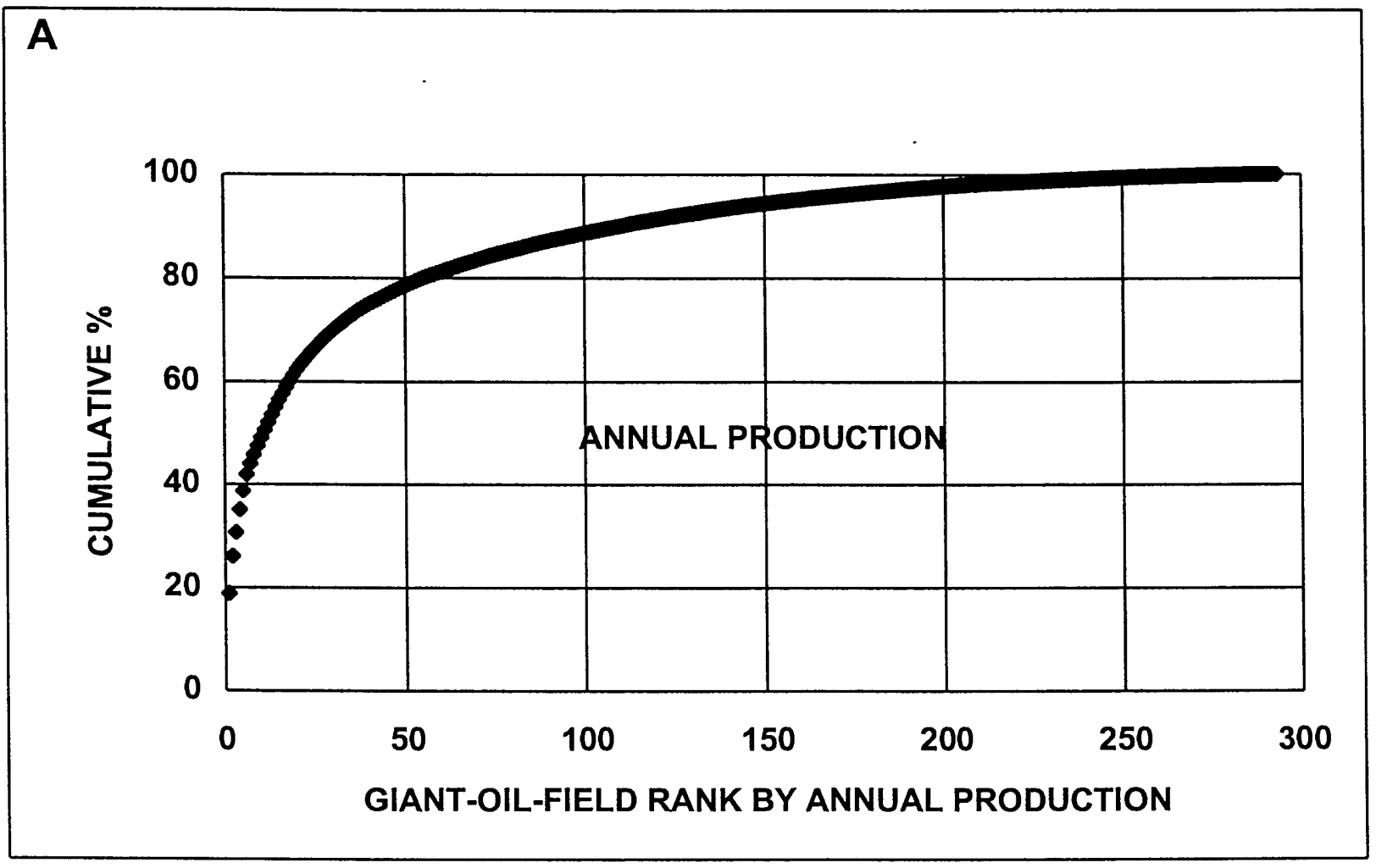

B

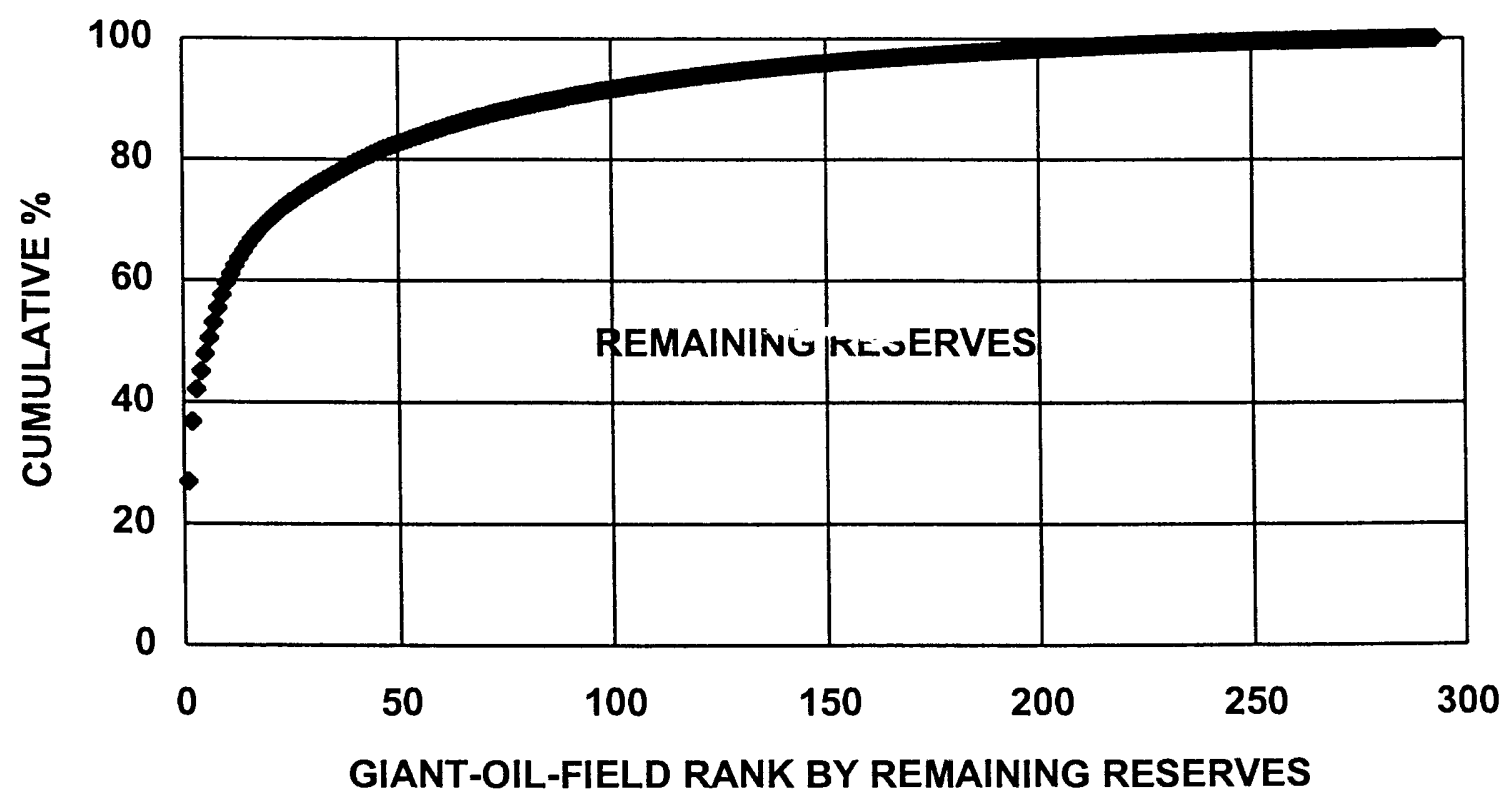

Figure 2. Cumulative percent of total annual production in 1997 (A) and of total remaining reserves as of 1/1/98 (B) for giant U.S. oil fields. Data are from Table 1. 


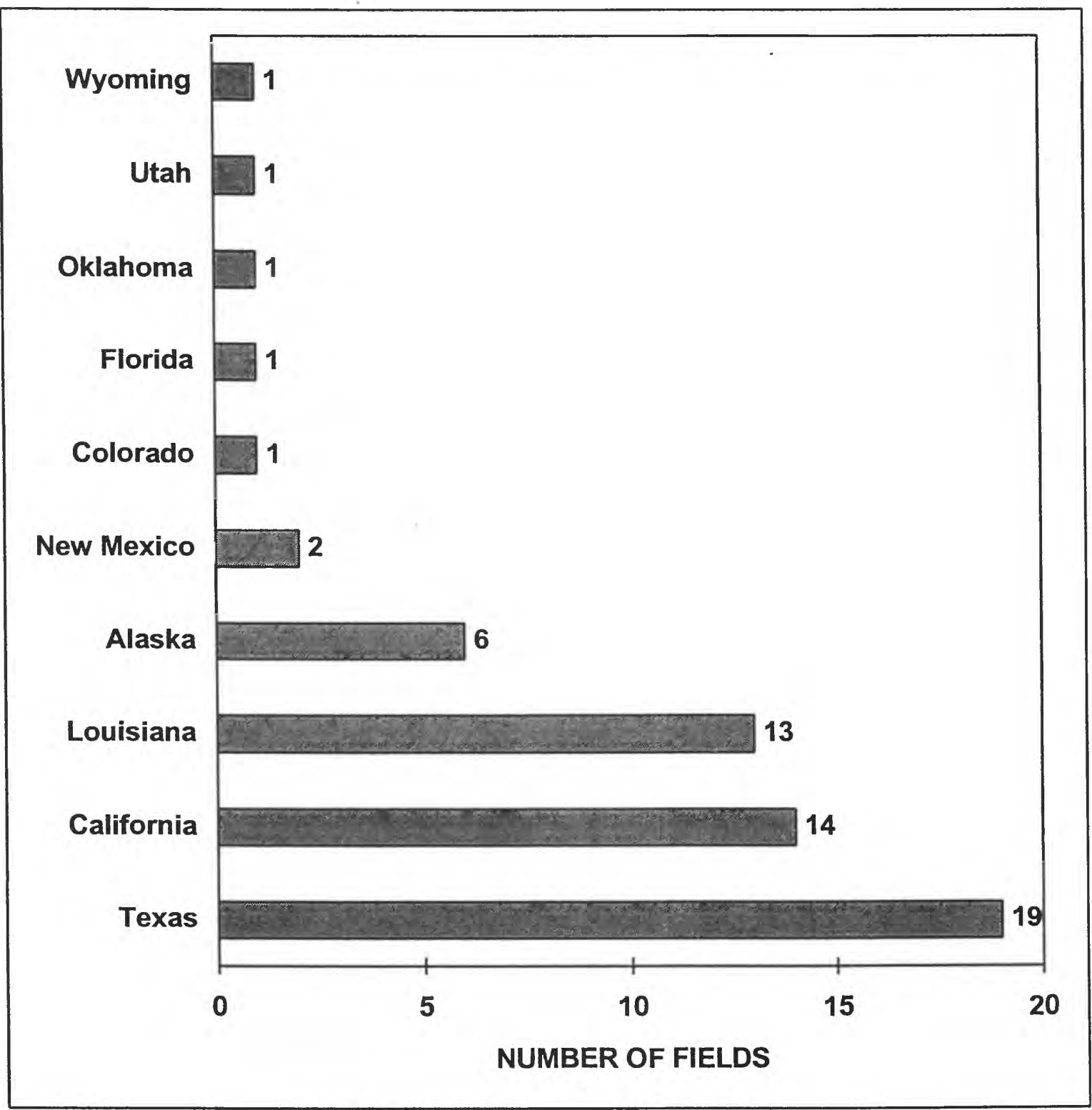

Figure 3. Locations of giant U.S. oil fields having significant remaining potential. These fields are defined and listed in Table 3. 

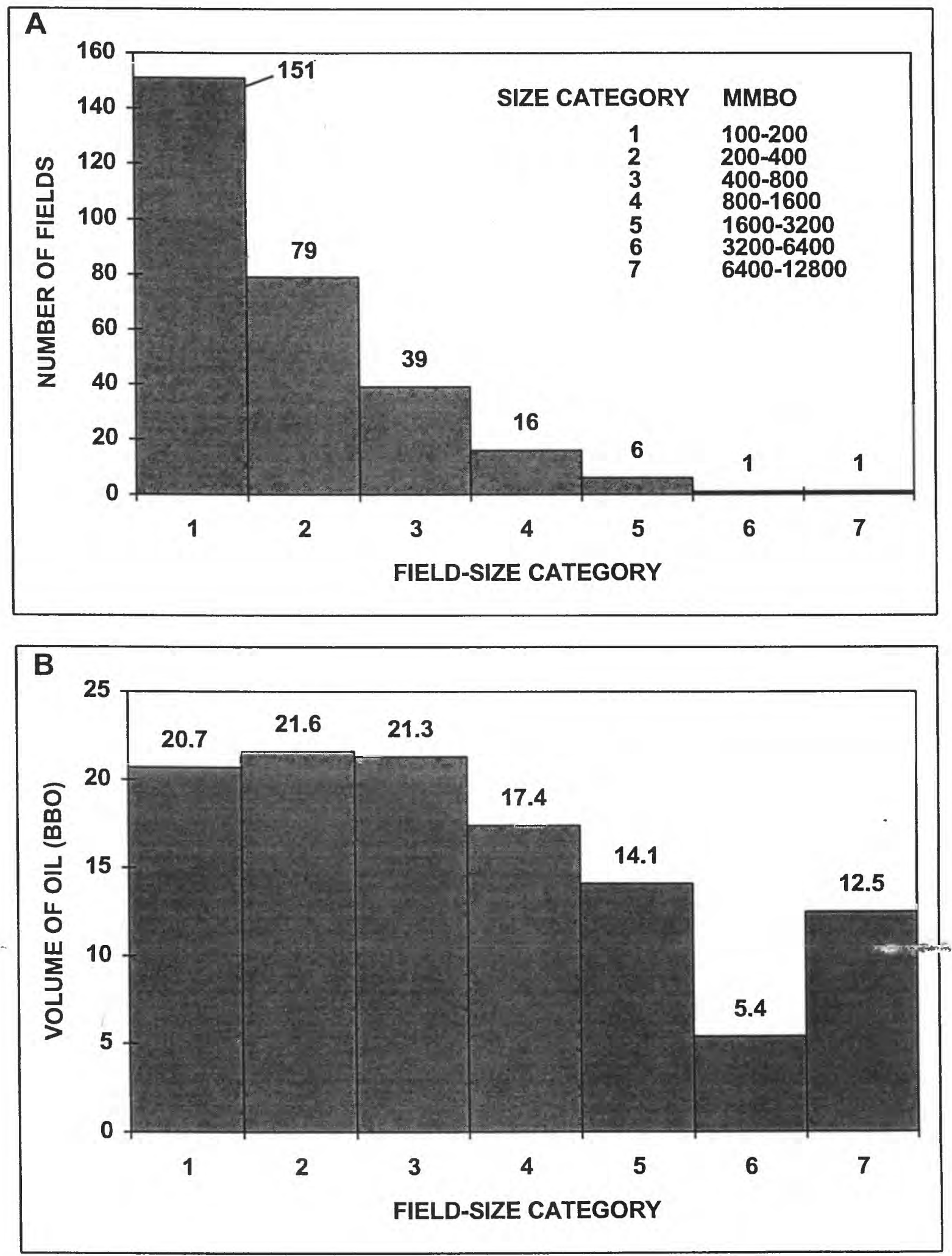

Figure 4. Number of fields (A) and volume of oil (estimated total recovery as of 1/1/98) (B) by field-size category, for giant U.S. oil fields. Data are from Table 1. 


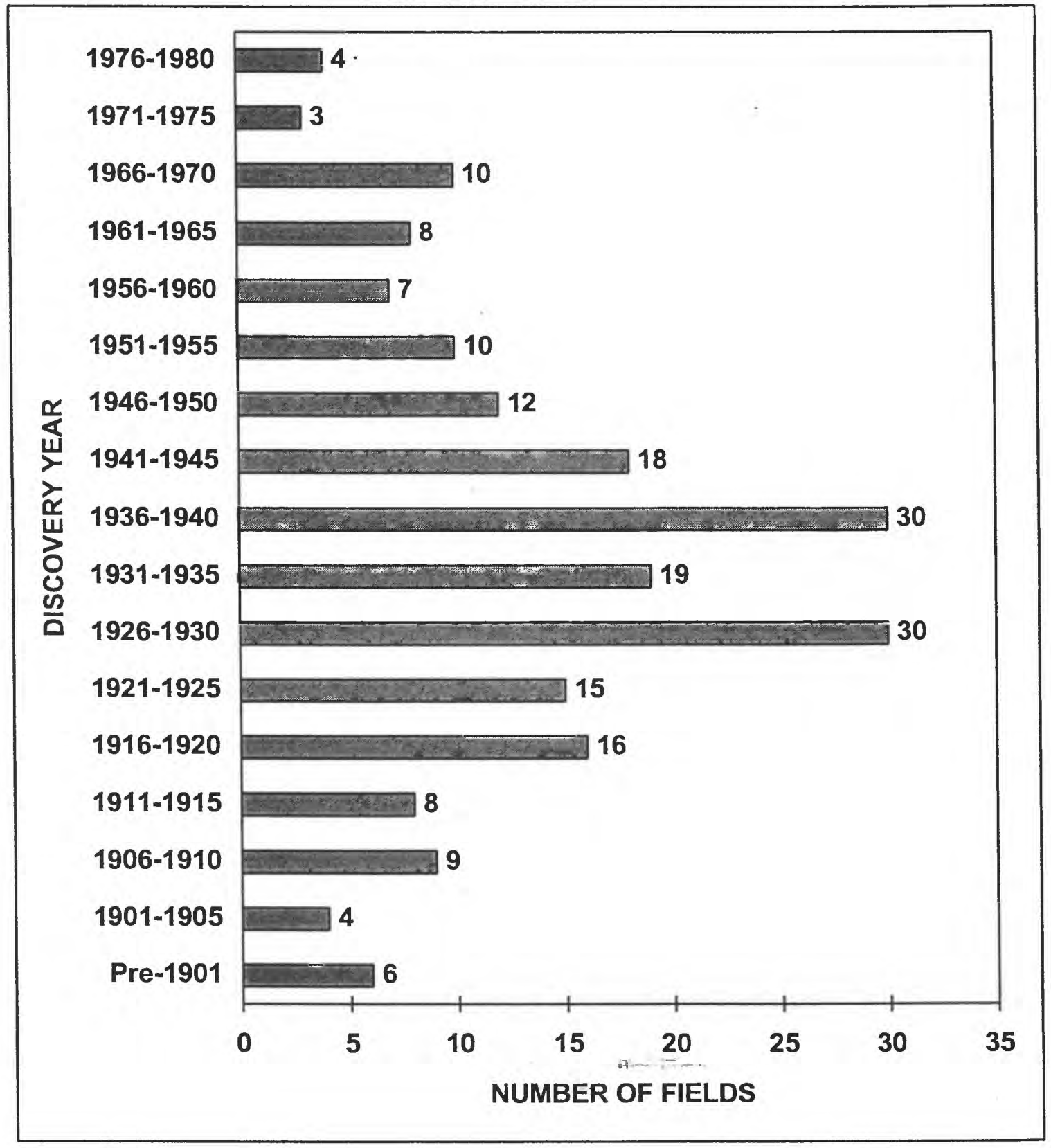

Figure 5. Discovery year of giant U.S. oil fields for which production and reserves data as of $1 / 1 / 90$ and 1/1/98 are available. Data are from Table 2. 

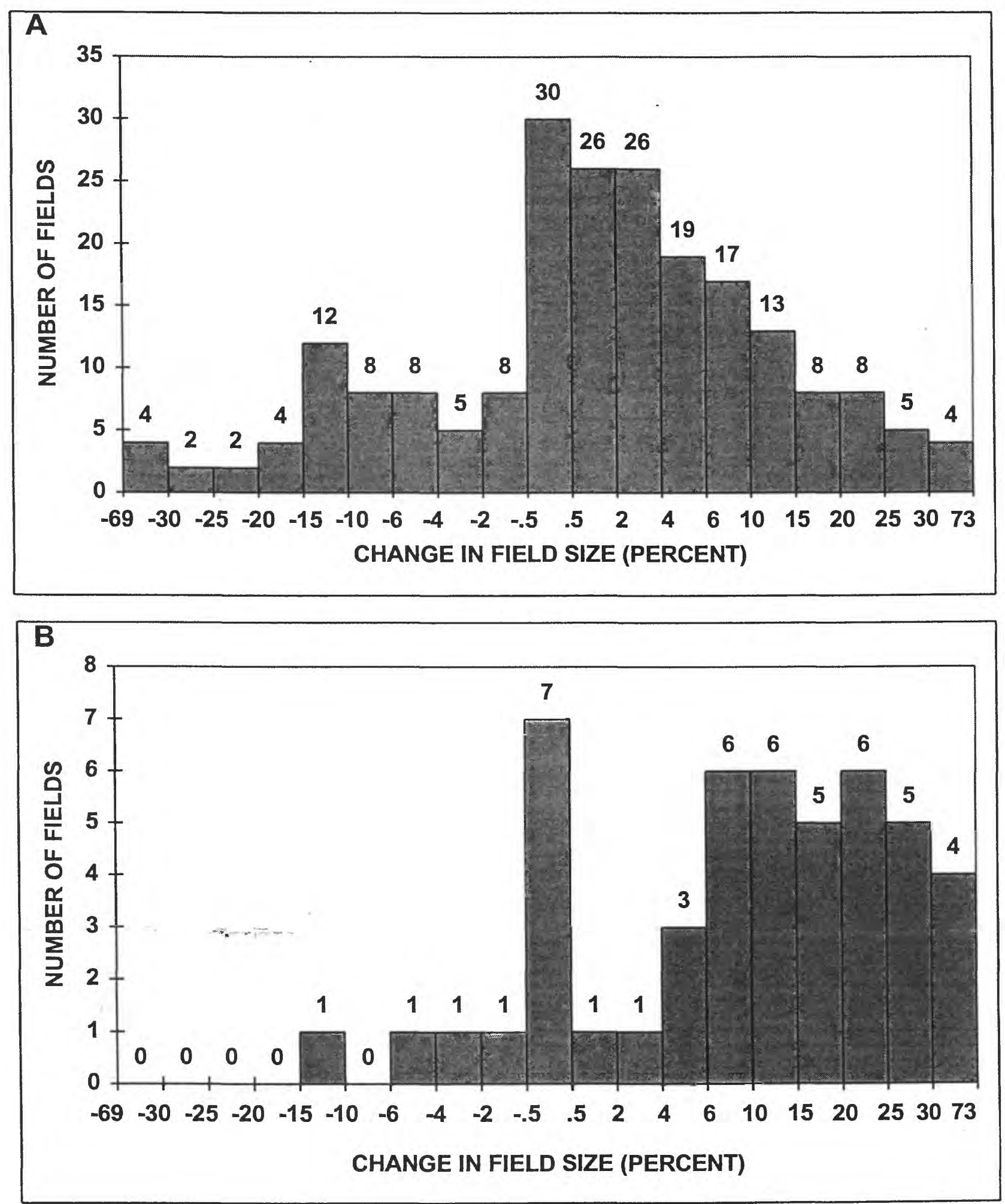

Figure 6. Number of giant U.S. oil fields (A) and number of giant U.S. oil fields having significant remaining potential $(B)$ by change in field size - measured in percent - from 1/1/90 to 1/1/98. Data are from Tables 2 and 3. 

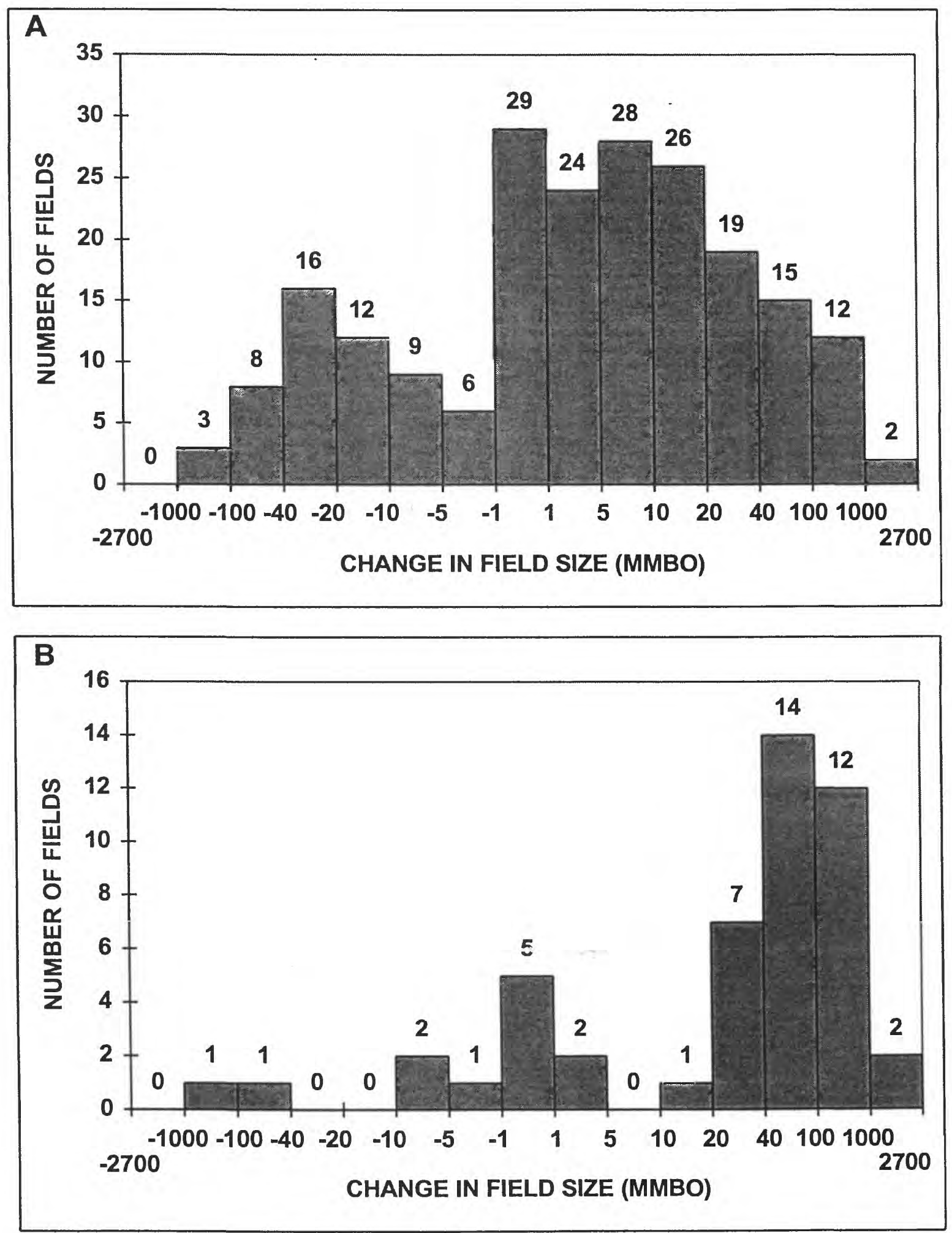

Figure 7. Number of giant U.S. oil fields (A) and number of giant U.S. oil fields having significant remaining potential $(B)$ by change in field size - measured in million barrels of oil - from 1/1/90 to 1/1/98. Data are from Tables 2 and 3. 


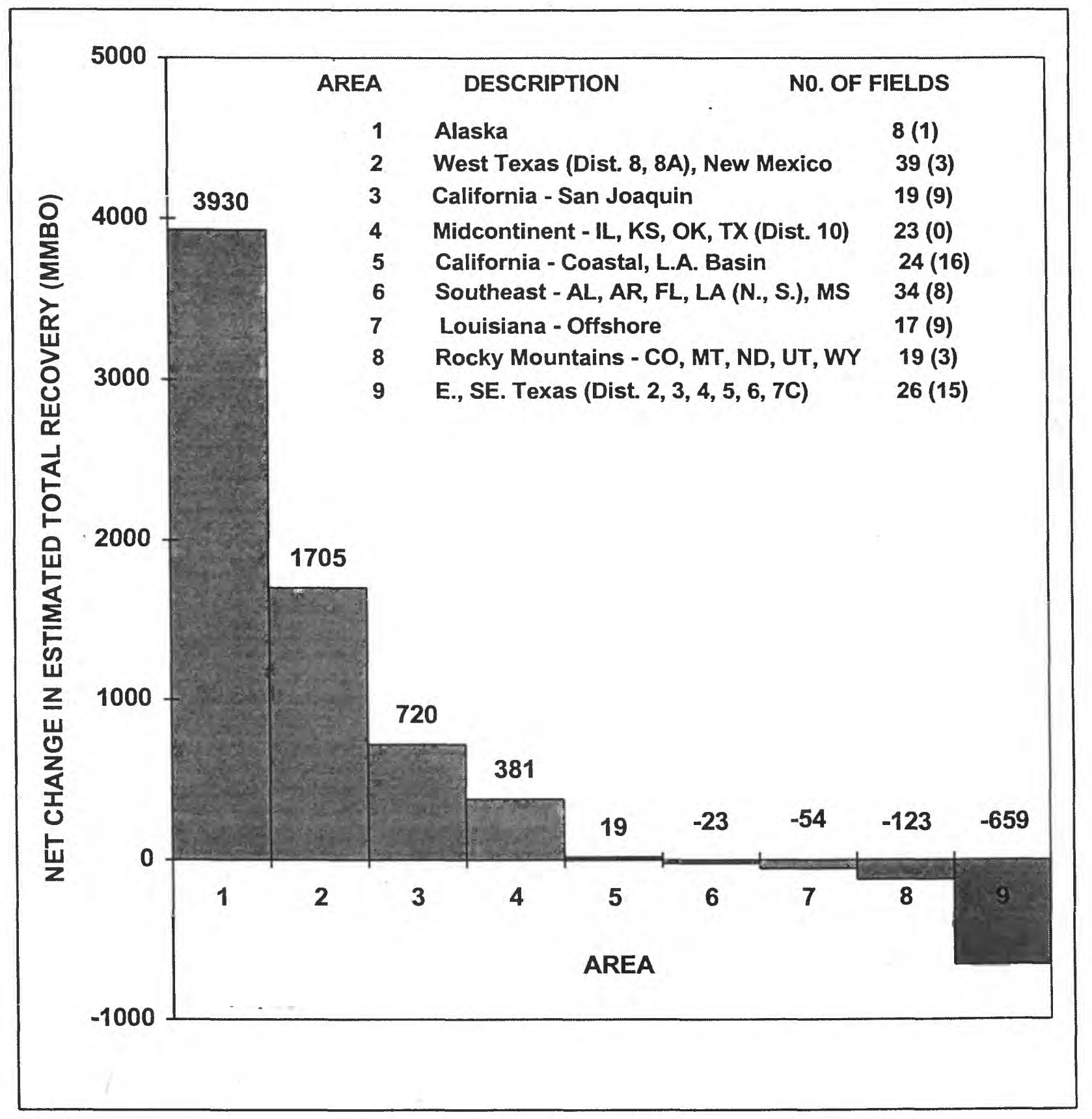

Figure 8. Net change in estimated total recovery from $1 / 1 / 90$ to $1 / 1 / 98$ by areas of the U.S., for giant U.S. oil fields. Data are from Table 2. Column for number of fields shows total number and number that declined in size (in parenthesis). 
Table 1. U.S. oil fields having estimated total recovery exceeding 100 million barrels of oil (mmbo). [Data are from International Petroleum Encyclopedia (1998). Production and reserves are in mmbo. Estimated total recovery = cumulative production plus remaining reserves. Res./Prod. = ratio of remaining reserves to 1997 production.]

\begin{tabular}{|c|c|c|c|c|c|c|c|}
\hline Field & Location & $\begin{array}{ll}\text { Discovery } & F \\
\text { Year } & \text { ir }\end{array}$ & $\begin{array}{l}\text { Production } \\
\text { in } 1997\end{array}$ & $\begin{array}{l}\text { Cum. Production } \\
\text { as of } 1 / 1 / 98\end{array}$ & $\begin{array}{l}\text { Rem. Reserves } \\
\text { as of } 1 / 1 / 98\end{array}$ & $\begin{array}{l}\text { Est. Total Recovery } \\
\text { as of } 1 / 1 / 98\end{array}$ & $\begin{array}{l}\text { Res./Prod. } \\
\text { (years) }\end{array}$ \\
\hline 1 Citronelle & Alabama & 1955 & 1.157 & 159.4 & 4.4 & 163.8 & 3.8 \\
\hline 2 Endicott & Alaska & 1978 & 21.000 & 358.0 & 170.0 & 528.0 & 8.1 \\
\hline 3 Granite Point & Alaska & 1965 & 2.000 & 130.0 & 16.0 & 146.0 & 8.0 \\
\hline 4 Kuparuk River & Alaska & 1969 & 96.000 & 1484.0 & 1111.0 & 2595.0 & 11.6 \\
\hline 5 Lisburne & Alaska & 1967 & 3.000 & 119.0 & 32.0 & 151.0 & 10.7 \\
\hline 6 McArthur River & Alaska & 1965 & 5.000 & 586.0 & 44.0 & 630.0 & 8.8 \\
\hline 7 Mid. Ground Shoal & Alaska & 1962 & 2.000 & 178.6 & 12.0 & 190.6 & 6.0 \\
\hline 8 Milne Point & Alaska & 1969 & 19.000 & 85.0 & 223.0 & 308.0 & 11.7 \\
\hline 9 Point Mclntyre & Alaska & 1988 & 59.000 & 212.0 & 241.0 & 453.0 & 4.1 \\
\hline 10 Prudhoe Bay & Alaska & 1968 & 251.000 & 9507.0 & 3042.0 & 12549.0 & 12.1 \\
\hline 11 Swanson River & Alaska & 1957 & 1.000 & 224.4 & 11.0 & 235.4 & 11.0 \\
\hline 12 Magnolia & Arkansas & 1938 & 0.330 & 165.8 & 2.6 & 168.4 & 7.9 \\
\hline 13 Smackover & Arkansas & 1922 & 1.570 & 574.4 & 4.7 & 579.1 & 3.0 \\
\hline 14 Belridge $N$. & Cal.-San Joaq. & 1912 & 1.649 & 106.4 & 10.1 & 116.5 & 6.1 \\
\hline 15 Belridge S. & Cal.-San Joaq. & 1911 & 42.848 & 1108.6 & 272.3 & 1380.9 & 6.4 \\
\hline 16 Buena Vista & Cal.-San Joaq. & 1909 & 0.886 & 659.2 & 18.5 & 677.7 & 20.9 \\
\hline 17 Coalinga & Cal.-San Joaq. & 1890 & 9.732 & 849.3 & 57.1 & 906.4 & 5.9 \\
\hline 18 Coalinga Nose & Cal.-San Joaq. & 1938 & 0.233 & 503.9 & 4.5 & 508.4 & 19.3 \\
\hline 19 Coles Levee N. & Cal.-San Joaq. & 1938 & 0.225 & 163.1 & 1.6 & 164.7 & 7.1 \\
\hline 20 Cuyama S. & Cal.-San Joaq. & 1949 & 0.254 & 221.5 & 3.5 & 225.0 & 13.8 \\
\hline 21 Cymric & Cal.-San Joaq. & 1909 & 13.847 & 294.2 & 68.6 & 362.8 & 5.0 \\
\hline 22 Edison & Cal.-San Joaq. & 1928 & 0.816 & 141.8 & 4.6 & 146.4 & 5.6 \\
\hline 23 Elk Hills & Cal.-San Joaq. & 1911 & 20.529 & 1119.3 & 288.6 & 1407.9 & 14.1 \\
\hline 24 Fruitvale & Cal.-San Joaq. & 1928 & 0.471 & 120.2 & 5.9 & 126.1 & 12.5 \\
\hline 25 Greeley & Cal.-San Joaq. & 1936 & 0.158 & 114.4 & 0.8 & 115.2 & 5.1 \\
\hline 26 Kern Front & Cal.-San Joaq. & 1912 & 2.047 & 193.3 & 23.6 & 216.9 & 11.5 \\
\hline 27 Kern River & Cal.-San Joaq. & 1899 & 48.746 & 1618.8 & 329.0 & 1947.8 & 6.7 \\
\hline 28 Kettleman N. Dome & Cal.-San Joaq. & 1928 & 0.113 & 458.0 & 2.3 & 460.3 & 20.4 \\
\hline 29 Lost Hills & Cal.-San Joaq. & 1910 & 11.520 & 266.2 & 51.9 & 318.1 & 4.5 \\
\hline 30 Midway-Sunset & Cal.-San Joaq. & 1894 & 60.692 & 2360.1 & 332.7 & 2692.8 & 5.5 \\
\hline 31 Mount Poso & Cal.-San Joaq. & 1926 & 1.075 & 291.5 & 30.4 & 321.9 & 28.3 \\
\hline 32 Rio Bravo & Cal.-San Joaq. & 1937 & 0.039 & 116.6 & 0.3 & 116.9 & 7.7 \\
\hline 33 Round Mountain & Cal.-San Joaq. & 1927 & 0.273 & 98.7 & 2.1 & 100.8 & 7.7 \\
\hline 34 Yowlumne & Cal.-San Joaq. & 1974 & 1.101 & 106.2 & 11.0 & 117.2 & 10.0 \\
\hline 35 Carpinteria & Cal.-Coastal & 1966 & 0.768 & 99.0 & 9.7 & 108.7 & 12.6 \\
\hline 36 Cat Canyon E., W. & Cal.-Coastal & 1908 & 0.919 & 298.0 & 22.0 & 320.0 & 23.9 \\
\hline 37 Dos Cuadras & Cal.-Coastal & 1969 & 2.712 & 241.8 & 15.0 & 256.8 & 5.5 \\
\hline 38 Hondo & Cal.-Coastal & 1969 & $16.49 \%$ & 157.8 & 121.4 & 279.2 & 7.4 \\
\hline 39 Orcutt & Cal.-Coastal & 1901 & 0.766 & 172.5 & 5.5 & 178.0 & 7.2 \\
\hline 40 Pescado & Cal.-Coastal & 1969 & 15.161 & 57.4 & 51.7 & 109.1 & 3.4 \\
\hline 41 Point Arguello & Cal.-Coastal & 1981 & 10.563 & 123.2 & 160.7 & 283.9 & 15.2 \\
\hline 42 Rincon & Cal.-Coastal & 1927 & 1.006 & 156.4 & 3.6 & 160.0 & 3.6 \\
\hline 43 San Ardo & Cal.-Coastal & 1947 & 4.629 & 447.2 & 82.8 & 530.0 & 17.9 \\
\hline 44 Santa Maria Valley & Cal.-Coastal & 1934 & 0.382 & 204.2 & 14.4 & 218.6 & 37.7 \\
\hline 45 South Mountain & Cal.-Coastal & 1916 & 0.730 & 152.4 & 4.6 & 157.0 & 6.3 \\
\hline 46 Ventura & Cal.-Coastal & 1919 & 5.191 & 945.3 & 66.8 & 1012.1 & 12.9 \\
\hline 47 Beta & Cal.-L.A. Basin & 1976 & 3.009 & 73.0 & 140.5 & 213.5 & 46.7 \\
\hline 48 Beverly Hills & Cal.-L.A. Basin & 1900 & 1.395 & 135.5 & 12.1 & 147.6 & 8.7 \\
\hline 49 Brea-Olinda & Cal.-L.A. Basin & 1880 & 1.382 & 398.4 & 40.3 & 438.7 & 29.2 \\
\hline 50 Coyote $\mathrm{E}$. & Cal.-L.A. Basin & 1909 & 0.381 & 112.7 & 9.1 & 121.8 & 23.9 \\
\hline 51 Dominguez & Cal.-L.A. Basin & 1923 & 0.348 & 273.7 & 3.1 & 276.8 & 8.9 \\
\hline 52 Huntington Beach & Cal.-L.A. Basin & 1920 & 3.682 & 1099.5 & 38.6 & 1138.1 & 10.5 \\
\hline 53 Inglewood & Cal.-L.A. Basin & 1924 & 2.457 & 365.6 & 26.7 & 392.3 & 10.9 \\
\hline 54 Long Beach & Cal.-L.A. Basin & 1921 & 1.683 & 926.0 & 18.6 & 944.6 & 11.1 \\
\hline 55 Montebello & Cal.-L.A. Basin & 1917 & 0.271 & 195.7 & 6.3 & 202.0 & 23.2 \\
\hline 56 Richfield & Cal.-L.A. Basin & 1919 & 0.399 & 197.6 & 5.3 & 202.9 & 13.3 \\
\hline 57 San Miguelito & Cal.-L.A. Basin & 1931 & 0.967 & 109.6 & 28.4 & 138.0 & 29.4 \\
\hline
\end{tabular}




\begin{tabular}{|c|c|c|c|c|c|c|}
\hline Field & Location & $\begin{array}{ll}\text { Discovery } & P \\
\text { Year } & \text { in }\end{array}$ & $\begin{array}{l}\text { Production C } \\
\text { in } 1997 \text { as }\end{array}$ & $\begin{array}{l}\text { Cum. Production } \\
\text { as of } 1 / 1 / 98\end{array}$ & $\begin{array}{l}\text { Rem. Reserves } \\
\text { as of } 1 / 1 / 98\end{array}$ & $\begin{array}{l}\text { Est. Total Recovery } \\
\text { as of } 1 / 1 / 98\end{array}$ \\
\hline 8 Santa Fe Springs & Cal.-L.A. Basin & 1919 & 0.732 & 619.9 & 12.8 & 632.7 \\
\hline 9 Seal Beach & Cal.-L.A. Basin & 1924 & 0.516 & 208.9 & 7.6 & 216.5 \\
\hline 0 Torrance & Cal.-L.A. Basin & 1922 & 0.789 & 220.9 & 18.0 & 238.9 \\
\hline 1 Yorba Linda & Cal.-L.A. Basin & 1930 & 0.449 & 91.1 & 10.2 & 101.3 \\
\hline 2 Wilmington & Cal.-L.A. Basin & 1932 & 19.779 & 2497.3 & 290.9 & 2788.2 \\
\hline 3 Rangely & Colorado & 1933 & 7.529 & 833.6 & 70.4 & 904.0 \\
\hline 4 Jay & Florida & 1970 & 3.777 & 395.4 & 23.1 & 418.5 \\
\hline 5 Clay City & Illinois & 1938 & 0.975 & 409.8 & 6.3 & 416.1 \\
\hline 6 Dale Consolidated & Illinois & 1940 & 0.077 & 105.5 & 0.7 & 106.2 \\
\hline 7 Lawrence & Illinois & 1906 & 1.743 & 414.8 & 5.3 & 420.1 \\
\hline 8 Louden & Illinois & 1938 & 0.885 & 396.6 & 3.6 & 400.2 \\
\hline 9 Main & Illinois & 1906 & 1.054 & 246.8 & 5.8 & 252.6 \\
\hline 0 New Harmony & Illinois & 1938 & 0.619 & 160.3 & 3.1 & 163.4 \\
\hline 1 Salem & Illinois & 1938 & 0.939 & 234.9 & 2.9 & 237.8 \\
\hline 2 Bemis-Shutts & Kansas & 1928 & 1.024 & 254.2 & 6.2 & 260.4 \\
\hline 3 Chase-Silica & Kansas & 1931 & 0.665 & 309.4 & 3.0 & 312.4 \\
\hline 4 El Dorado & Kansas & 1917 & 0.712 & 306.0 & 4.1 & 310.1 \\
\hline 5 Hall-Gurney & Kansas & 1931 & 0.687 & 154.0 & 3.8 & 157.8 \\
\hline 6 Kraft-Prusa & Kansas & 1937 & 0.351 & 131.6 & 2.2 & 133.8 \\
\hline 77 Trapp & Kansas & 1937 & 0.665 & 234.2 & 3.6 & 237.8 \\
\hline 8 Bay Marchand 2 & Louisiana-Off. & 1949 & 5.311 & 695.2 & 40.9 & 736.1 \\
\hline 79 Breton Sound 20 & Louisiana-Off. & 1953 & 2.002 & 114.2 & 8.8 & 123.0 \\
\hline 30 Eugene Island 126 & Louisiana-Off. & 1950 & 0.689 & 122.6 & 5.1 & 127.7 \\
\hline 31 Eugene Island 175 & Louisiana-Off. & 1956 & 3.296 & 95.4 & 15.9 & 111.3 \\
\hline 32 Eugene Island 276 & Louisiana-Off. & 1964 & 1.809 & 89.1 & 15.6 & 104.7 \\
\hline 33 Eugene Island 330 & Louisiana-Off. & 1930 & 8.655 & 341.3 & 47.5 & 388.8 \\
\hline 34 Garden Banks 426 & Louisiana-Off. & 1987 & 22.706 & 73.1 & 93.4 & 166.5 \\
\hline 35 Grand Isle 16 & Louisiana-Off. & 1948 & 2.659 & 285.9 & 18.4 & 304.3 \\
\hline 36 Grand Isle 43 & Louisiana-Off. & 1956 & 2.884 & 302.8 & 48.1 & 350.9 \\
\hline 87 Grand Isle 47 & Louisiana-Off. & 1955 & 1.412 & 94.6 & 21.8 & 116.4 \\
\hline 88 Green Canyon 65 & Louisiana-Off. & 1984 & 7.133 & 93.5 & 46.5 & 140.0 \\
\hline 89 Main Pass 35 & Louisiana-Off. & 1951 & 0.450 & 99.4 & 3.8 & 103.2 \\
\hline 90 Main Pass 41 & Louisiana-Off. & 1957 & 3.841 & 258.5 & 17.7 & 276.2 \\
\hline 91 Main Pass 69 & Louisiana-Off. & 1948 & 1.959 & 261.9 & 13.0 & 274.9 \\
\hline 92 Main Pass 144 & Louisiana-Off. & 1967 & 1.614 & 113.7 & 12.4 & 126.1 \\
\hline 93 Main Pass 299 & Louisiana-Off. & 1962 & 6.903 & 109.2 & 45.1 & 154.3 \\
\hline 94 Main Pass 306 & Louisiana-Off. & 1969 & 0.979 & 212.4 & 5.4 & 217.8 \\
\hline 95 Main Pass 311 & Louisiana-Off. & 1977 & 2.860 & 76.6 & 27.2 & 103.8 \\
\hline 96 Miss. Canyon 194 & Louisiana-Off. & 1980 & 3.213 & 161.0 & 59.0 & 220.0 \\
\hline 97 Ship Shoal 113 & Louisiana-Off. & 1955 & 2.019 & 100.4 & 24.1 & 124.5 \\
\hline 98 Ship Shoal 207 & Louisiana-Off. & 1967 & 0.510 & 94.8 & 6.0 & 100.8 \\
\hline 99 Ship Shoal 208 & Louisiana-Off. & 1962 & $2.17 u$ & 184.1 & 12.7 & 196.8 \\
\hline 00 S. Marsh Island 128 & Louisiana-Off. & 1974 & 1.405 & 114.3 & 8.9 & 123.2 \\
\hline 01 South Pass 24 & Louisiana-Off. & 1950 & 4.850 & 284.7 & 27.9 & 312.6 \\
\hline 02 South Pass 27 & Louisiana-Off. & 1954 & 2.402 & 152.1 & 13.4 & 165.5 \\
\hline 03 South Pass 61 & Louisiana-Off. & 1968 & 7.914 & 203.1 & 29.2 & 232.3 \\
\hline 04 South Pass 62 & Louisiana-Off. & 1965 & 2.188 & 127.9 & 12.5 & 140.4 \\
\hline 05 South Pass 65 & Louisiana-Off. & 1965 & 1.440 & 121.0 & 9.2 & 130.2 \\
\hline 06 South Pass 89 & Louisiana-Off. & 1969 & 7.290 & 154.1 & 41.6 & 195.7 \\
\hline 07 S. Timbalier 21 & Louisiana-Off. & 1939 & 1.643 & 234.5 & 8.4 & 242.9 \\
\hline 08 West Delta 30 & Louisiana-Off. & 1949 & 4.779 & 496.8 & 29.6 & 526.4 \\
\hline 09 West Delta 73 & Louisiana-Off. & 1962 & 3.803 & 230.4 & 49.6 & 280.0 \\
\hline 10 West Delta 79 & Louisiana-Off. & 1966 & 0.844 & 148.0 & 11.0 & 159.0 \\
\hline 11 Avery Island & Louisiana-S. & 1942 & 0.611 & 100.0 & 2.6 & 102.6 \\
\hline 12 Bay de Chene & Louisiana-S. & 1941 & 0.331 & 99.1 & 2.3 & 101.4 \\
\hline 13 Bay St. Elaine & Louisiana-S. & 1928 & 0.222 & 167.5 & 1.0 & 168.5 \\
\hline 14 Bayou Sale & Louisiana-S. & 1941 & 0.337 & 165.4 & 1.3 & 166.7 \\
\hline 15 Black Bay W. & Louisiana-S. & 1953 & 1.214 & 159.6 & 4.6 & 164.2 \\
\hline 16 Caillou Island & Louisiana-S. & 1930 & 2.882 & 625.3 & 16.4 & 641.7 \\
\hline 17 Cote Blanche Bay & Louisiana-S. & 1940 & 0.318 & 188.2 & 2.8 & 191.0 \\
\hline 18 Cote Blanche Island & Louisiana-S. & 1948 & 1.315 & 188.5 & 5.3 & 193.8 \\
\hline
\end{tabular}




\begin{tabular}{|c|c|c|c|c|c|c|c|}
\hline Field & Location & $\begin{array}{l}\text { Discovery } \\
\text { Year }\end{array}$ & $\begin{array}{l}\text { Production } \\
\text { in } 1997\end{array}$ & $\begin{array}{l}\text { Cum. Production } F \\
\text { as of } 1 / 1 / 98\end{array}$ & $\begin{array}{l}\text { Rem. Reserves } \\
\text { as of } 1 / 1 / 98\end{array}$ & $\begin{array}{l}\text { Est. Total Recovery } \\
\text { as of } 1 / 1 / 98\end{array}$ & $\begin{array}{l}\text { Res./Prod. } \\
\text { (years) }\end{array}$ \\
\hline 119 Delta Farms & Louisiana-S. & 1944 & 0.271 & 120.3 & 2.2 & 122.5 & 8.1 \\
\hline 120 Garden Island Bay & Louisiana-S. & 1934 & 1.107 & 230.7 & 3.8 & 234.5 & 3.4 \\
\hline 121 Golden Meadow & Louisiana-S. & 1938 & 0.320 & 139.0 & 1.2 & 140.2 & 3.8 \\
\hline 122 Grand Bay & Louisiana-S. & 1938 & 0.559 & 177.4 & 2.0 & 179.4 & 3.6 \\
\hline 123 Hackberry E. & Louisiana-S. & 1927 & 0.317 & 112.7 & 4.0 & 116.7 & 12.6 \\
\hline 124 Hackberry W. & Louisiana-S. & 1928 & 0.398 & 148.8 & 2.1 & 150.9 & 5.3 \\
\hline 125 Iberia & Louisiana-S. & 1917 & 0.235 & 99.9 & 0.8 & 100.7 & 3.4 \\
\hline 126 Jennings & Louisiana-S. & 1901 & 0.137 & 118.2 & 1.2 & 119.4 & 8.8 \\
\hline 127 Lafitte & Louisiana-S. & 1935 & 0.633 & 264.7 & 3.5 & 268.2 & 5.5 \\
\hline 128 Lake Barre & Louisiana-S. & 1929 & 2.095 & 214.8 & 11.9 & 226.7 & 5.7 \\
\hline 129 Lake Washington & Louisiana-S. & 1931 & 1.694 & 260.2 & 6.7 & 266.9 & 4.0 \\
\hline 130 Leeville & Louisiana-S. & 1931 & 0.313 & 145.9 & 3.0 & 148.9 & 9.6 \\
\hline 131 Quarantine Bay & Louisiana-S. & 1937 & 0.888 & 179.9 & 6.2 & 186.1 & 7.0 \\
\hline 132 Romere Pass & Louisiana-S. & 1950 & 0.301 & 100.9 & 1.6 & 102.5 & 5.3 \\
\hline 133 Timbalier Bay onsh. & Louisiana-S. & 1938 & 0.795 & 306.6 & 12.6 & 319.2 & 15.8 \\
\hline 134 Venice & Louisiana-S. & 1937 & 0.200 & 186.1 & 3.4 & 189.5 & 17.0 \\
\hline 135 Vinton & Louisiana-S. & 1910 & 0.172 & 163.4 & 1.8 & 165.2 & 10.5 \\
\hline 136 Weeks Island & Louisiana-S. & 1945 & 3.011 & 246.3 & 13.0 & 259.3 & 4.3 \\
\hline 137 West Bay & Louisiana-S. & 1940 & 0.903 & 237.1 & 7.5 & 244.6 & 8.3 \\
\hline 138 White Castle & Louisiana-S. & 1929 & 0.227 & 93.5 & 6.6 & 100.1 & 29.1 \\
\hline 139 Caddo-Pine Island & Louisiana-N. & 1905 & 1.899 & 383.6 & 10.9 & 394.5 & 5.7 \\
\hline 140 Delhi & Louisiana-N. & 1944 & 0.033 & 213.5 & 2.9 & 216.4 & 87.9 \\
\hline 141 Haynesville & Louisiana-N. & 1921 & 0.630 & 177.6 & 3.8 & 181.4 & 6.0 \\
\hline 142 Homer & Louisiana-N. & 1919 & 0.286 & 101.4 & 1.3 & 102.7 & 4.5 \\
\hline 143 Nebo-Hemphill & Louisiana-N. & 1940 & 1.551 & 102.8 & 8.7 & 111.5 & 5.6 \\
\hline 144 Rodessa & Louisiana-N. & 1935 & 0.211 & 109.1 & 1.4 & 110.5 & 6.6 \\
\hline 145 Baxterville & Mississippi & 1944 & 0.937 & 252.2 & 5.2 & 257.4 & 5.5 \\
\hline 146 Heidelberg & Mississippi & 1944 & 1.996 & 196.0 & 5.6 & 201.6 & 2.8 \\
\hline 147 Tinsley & Mississippi & 1939 & 0.338 & 224.8 & 1.3 & 226.1 & 3.8 \\
\hline 148 Cabin Creek & Montana & 1953 & 1.214 & 102.2 & 6.8 & 109.0 & 5.6 \\
\hline 149 Cut Bank & Montana & 1926 & 0.459 & 168.2 & 2.0 & 170.2 & 4.4 \\
\hline 150 Elk Basin & Montana & 1915 & 0.444 & 100.1 & 1.3 & 101.4 & 2.9 \\
\hline 151 Pennel & Montana & 1955 & 1.905 & 95.5 & 11.4 & 106.9 & 6.0 \\
\hline 152 Pine & Montana & 1951 & 0.757 & 113.7 & 3.3 & 117.0 & 4.4 \\
\hline 153 Blinebry-Drinkard & New Mexico & 1945 & 0.959 & 142.2 & 4.1 & 146.3 & 4.3 \\
\hline 154 Denton & New Mexico & 1949 & 0.519 & 145.2 & 2.1 & 147.3 & 4.0 \\
\hline 155 Empire-Abo & New Mexico & 1957 & 0.556 & 228.3 & 2.9 & 231.2 & 5.2 \\
\hline 156 Eunice-Monument & New Mexico & 1929 & 2.387 & 147.4 & 6.6 & 154.0 & 2.8 \\
\hline 157 Grayburg-Jackson & New Mexico & 1929 & 4.059 & 118.5 & 18.3 & 136.8 & 4.5 \\
\hline 158 Hobbs & New Mexico & 1928 & 2.949 & 339.4 & 17.7 & 357.1 & 6.0 \\
\hline 159 Langlie-Mattix & New Mexico & 1935 & 0.944 & 135.1 & 4.6 & 139.7 & 4.9 \\
\hline 160 Maljamar & $\therefore \ldots$ Mexico & 1926 & 1.535 & 158.6 & 7.3 & 165.9 & 4.8 \\
\hline 161 Vacuum & New Mexico & 1929 & 8.015 & 508.5 & 31.2 & 539.7 & 3.9 \\
\hline 162 Beaver Lodge & N. Dakota & 1951 & 0.988 & 123.5 & 5.1 & 128.6 & 5.2 \\
\hline 163 Bowlegs & Oklahoma & 1927 & 0.071 & 163.7 & 1.1 & 164.8 & 15.5 \\
\hline 164 Burbank & Oklahoma & 1920 & 0.672 & 543.7 & 2.8 & 546.5 & 4.2 \\
\hline 165 Cement & Oklahoma & 1917 & 1.077 & 143.1 & 5.9 & 149.0 & 5.5 \\
\hline 166 Cushing & Oklahoma & 1912 & 1.037 & 508.6 & 5.8 & 514.4 & 5.6 \\
\hline 167 Earlsboro & Oklahoma & 1926 & 0.087 & 203.5 & 0.8 & 204.3 & 9.2 \\
\hline 168 Eola-Robberson & Oklahoma & 1921 & 0.374 & 139.9 & 1.5 & 141.4 & 4.0 \\
\hline 169 Fitts & Oklahoma & 1933 & 1.820 & 219.6 & 8.3 & 227.9 & 4.6 \\
\hline 170 Glen Pool & Oklahoma & 1905 & 0.413 & 333.9 & 4.6 & 338.5 & 11.1 \\
\hline 171 Golden Trend & Oklahoma & 1945 & 2.366 & 502.3 & 21.0 & 523.3 & 8.9 \\
\hline 172 Healdton & Oklahoma & 1913 & 1.279 & 348.7 & 4.2 & 352.9 & 3.3 \\
\hline$\uparrow 73$ Hewitt & Oklahoma & 1919 & 1.618 & 285.7 & 5.4 & 291.1 & 3.3 \\
\hline 174 Little River & Oklahoma & 1927 & 0.089 & 142.5 & 1.8 & 144.3 & 20.2 \\
\hline 175 Oklahoma City & Oklahoma & 1928 & 0.418 & 823.3 & 5.8 & 829.1 & 13.9 \\
\hline 176 Postle & Oklahoma & 1960 & 1.489 & 115.9 & 6.4 & 122.3 & 4.3 \\
\hline 177 Seminole & Oklahoma & 1926 & 0.125 & 195.0 & 1.0 & 196.0 & 8.0 \\
\hline 178 Sho-Vel-Tum & Oklahoma & 1919 & 10.800 & 1298.2 & 52.1 & 1350.3 & 4.8 \\
\hline 179 Sooner Trend & Oklahoma & 1965 & 2.714 & 327.6 & 16.9 & 344.5 & 6.2 \\
\hline
\end{tabular}


Discovery Production Cum. Production Rem. Reserves Est. Total Recovery Res./Prod. Year in 1997 as of $1 / 1 / 98$ as of $1 / 1 / 98$ as of $1 / 1 / 98$ (years)

\begin{tabular}{|c|c|c|c|c|c|c|c|}
\hline 180 St. Louis & Oklahoma & 1925 & 0.388 & 181.1 & 1.4 & 182.5 & 3.6 \\
\hline 181 Darst Creek & Texas-Dist. 1 & 1929 & 0.551 & 159.2 & 3.1 & 162.3 & 5.6 \\
\hline 182 Luling-Branyon & Texas-Dist. 1 & 1922 & 0.929 & 155.7 & 3.4 & 159.1 & 3.7 \\
\hline 183 Pearsall & Texas-Dist. 1 & 1936 & 1.805 & 153.3 & 29.7 & 183.0 & 16.5 \\
\hline 184 Greta & Texas-Dist. 2 & 1933 & 0.277 & 150.9 & 1.6 & 152.5 & 5.8 \\
\hline 185 Lake Pasture & Texas-Dist. 2 & 1953 & 0.950 & 100.1 & 3.9 & 104.0 & 4.1 \\
\hline 186 Tom O'Connor & Texas-Dist. 2 & 1934 & 2.895 & 787.7 & 15.1 & 802.8 & 5.2 \\
\hline 187 West Ranch & Texas-Dist. 2 & 1938 & 0.789 & 391.9 & 5.2 & 397.1 & 6.6 \\
\hline 188 Anahuac & Texas-Dist. 3 & 1935 & 0.153 & 288.2 & 1.2 & 289.4 & 7.8 \\
\hline 189 Barbers Hill & Texas-Dist. 3 & 1916 & 0.185 & 113.5 & 0.8 & 114.3 & 4.3 \\
\hline 190 Conroe & Texas-Dist. 3 & 1931 & 1.160 & 742.4 & 4.9 & 747.3 & 4.2 \\
\hline 191 Giddings & Texas-Dist. 3 & 1971 & 16.590 & 457.0 & 66.9 & 523.9 & 4.0 \\
\hline 192 Goose Creek & Texas-Dist. 3 & 1906 & 0.488 & 122.9 & 1.8 & 124.7 & 3.7 \\
\hline 193 Hastings $E$. & Texas-Dist. 3 & 1934 & 1.061 & 707.0 & 3.2 & 710.2 & 3.0 \\
\hline 194 High Island & Texas-Dist. 3 & 1922 & 1.062 & 140.3 & 3.0 & 143.3 & 2.8 \\
\hline 195 Hull & Texas-Dist. 3 & 1918 & 0.411 & 171.7 & 2.2 & 173.9 & 5.4 \\
\hline 196 Humble & Texas-Dist. 3 & 1905 & 0.215 & 99.7 & 1.1 & 100.8 & 5.1 \\
\hline 197 Magnet Withers & Texas-Dist. 3 & 1936 & 0.916 & 121.3 & 5.5 & 126.8 & 6.0 \\
\hline 198 Manvel & Texas-Dist. 3 & 1931 & 0.315 & 103.5 & 1.4 & 104.9 & 4.4 \\
\hline 199 Oyster Bayou & Texas-Dist. 3 & 1941 & 0.339 & 164.6 & 1.5 & 166.1 & 4.4 \\
\hline 200 Raccoon Bend & Texas-Dist. 3 & 1927 & 0.369 & 104.8 & 1.5 & 106.3 & 4.1 \\
\hline 201 Sour Lake & Texas-Dist. 3 & 1902 & 0.494 & 102.7 & 7.0 & 109.7 & 14.2 \\
\hline 202 Thompson & Texas-Dist. 3 & 1921 & 1.102 & 487.9 & 5.7 & 493.6 & 5.2 \\
\hline 203 Tomball & Texas-Dist. 3 & 1933 & 0.188 & 123.1 & 1.0 & 124.1 & 5.3 \\
\hline 204 Webster & Texas-Dist. 3 & 1937 & 1.925 & 596.2 & 9.2 & 605.4 & 4.8 \\
\hline 205 W. Columbia & Texas-Dist. 3 & 1917 & 0.150 & 102.5 & 2.0 & 104.5 & 13.3 \\
\hline 206 Agua Dulce-Stratton & Texas-Dist. 4 & 1928 & 0.212 & 148.3 & 1.4 & 149.7 & 6.6 \\
\hline 207 Borregos & Texas-Dist. 4 & 1945 & 0.031 & 114.9 & 0.6 & 115.5 & 19.4 \\
\hline 208 Kelsey & Texas-Dist. 4 & 1938 & 0.052 & 116.1 & 0.8 & 116.9 & 15.4 \\
\hline 209 Plymouth & Texas-Dist. 4 & 1925 & 0.075 & 124.2 & 1.9 & 126.1 & 25.3 \\
\hline 210 Seeligson & Texas-Dist. 4 & 1925 & 0.081 & 272.8 & 0.7 & 273.5 & 8.6 \\
\hline 211 TCB & Texas-Dist. 4 & 1944 & 0.040 & 113.6 & 0.6 & 114.2 & 15.0 \\
\hline 212 White Point E. & Texas-Dist. 4 & 1938 & 0.022 & 104.7 & 0.6 & 105.3 & 27.3 \\
\hline 213 Van & Texas-Dist. 5 & 1928 & 1.559 & 541.7 & 8.3 & 550.0 & 5.3 \\
\hline 214 East Texas & Texas-Dist. 6 & 1930 & 19.213 & 5271.4 & 110.2 & 5381.6 & 5.7 \\
\hline 215 Fairway & Texas-Dist. 6 & 1960 & 1.825 & 210.1 & 12.8 & 222.9 & 7.0 \\
\hline 216 Hawkins & Texas-Dist. 6 & 1940 & 3.158 & 865.2 & 20.4 & 885.6 & 6.5 \\
\hline 217 Neches & Texas-Dist. 6 & 1953 & 0.431 & 110.1 & 1.6 & 111.7 & 3.7 \\
\hline 218 Quitman & Texas-Dist. 6 & 1948 & 0.605 & 129.6 & 2.7 & 132.3 & 4.5 \\
\hline 219 Talco & Texas-Dist. 6 & 1931 & 0.833 & 284.9 & 6.5 & 291.4 & 7.8 \\
\hline 220 Stephens Cnty Reg. & Texas-Dist. 7B & 1916 & 3298 & 172.7 & 15.7 & 188.4 & 4.8 \\
\hline 221 Big Lake & Texas-Dist. 7C & 1923 & 0.373 & 133.6 & 1.4 & 135.0 & 3.8 \\
\hline 222 McCamey & Texas-Dist. 7C & 1925 & 0.130 & 126.3 & 0.7 & 127.0 & 5.4 \\
\hline 223 Pegasus & Texas-Dist. 7C & 1949 & 0.814 & 143.4 & 3.7 & 147.1 & 4.5 \\
\hline 224 Andector & Texas-Dist. 8 & 1946 & 0.465 & 186.3 & 2.4 & 188.7 & 5.2 \\
\hline 225 Block 31 & Texas-Dist. 8 & 1945 & 1.114 & 238.6 & 7.8 & 246.4 & 7.0 \\
\hline 226 Cowden $N$ & Texas-Dist. 8 & 1930 & 9.595 & 598.3 & 50.5 & 648.8 & 5.3 \\
\hline 227 Cowden S.-Foster-J. & Texas-Dist. 8 & 1930 & 5.015 & 560.9 & 28.4 & 589.3 & 5.7 \\
\hline 228 Dollarhide & Texas-Dist. 8 & 1945 & 2.880 & 219.0 & 17.8 & 236.8 & 6.2 \\
\hline 229 Dune & Texas-Dist. 8 & 1938 & 1.149 & 199.4 & 6.3 & 205.7 & 5.5 \\
\hline 230 Fuhrman-Mascho & Texas-Dist. 8 & 1930 & 1.201 & 117.1 & 3.2 & 120.3 & 2.7 \\
\hline 231 Fullerton & Texas-Dist. 8 & 1942 & 6.655 & 412.1 & 35.2 & 447.3 & 5.3 \\
\hline 232 Goidsmith & Texas-Dist. 8 & 1934 & 4.198 & 799.3 & 26.9 & 826.2 & 6.4 \\
\hline 233 Hendrick & Texas-Dist. 8 & 1926 & 0.357 & 256.4 & 1.8 & 258.2 & 5.0 \\
\hline 234 Howard-Glasscock & Texas-Dist. 8 & 1925 & 4.215 & 447.9 & 20.8 & 468.7 & 4.9 \\
\hline 235 latan $E$ & Texas-Dist. 8 & 1926 & 2.137 & 165.3 & 12.7 & 178.0 & 5.9 \\
\hline 236 Jordan & Texas-Dist. 8 & 1937 & 0.611 & 135.5 & 3.0 & 138.5 & 4.9 \\
\hline 237 Kermit & Texas-Dist. 8 & 1928 & 0.271 & 116.5 & 1.6 & 118.1 & 5.9 \\
\hline 238 Keystone & Texas-Dist. 8 & 1930 & 1.414 & 325.7 & 5.5 & 331.2 & 3.9 \\
\hline 239 Mabee & Texas-Dist. 8 & 1943 & 2.440 & 108.1 & 13.7 & 121.8 & 5.6 \\
\hline 240 McElroy & Texas-Dist. 8 & 1926 & 7.455 & 540.1 & 36.4 & 576.5 & 4.9 \\
\hline
\end{tabular}




\begin{tabular}{|c|c|c|c|c|c|c|c|}
\hline Field & Location & $\begin{array}{ll}\text { Discovery } & F \\
\text { Year } & \text { ir }\end{array}$ & $\begin{array}{l}\text { Production } \\
\text { in } 1997\end{array}$ & $\begin{array}{l}\text { Cum. Production } \\
\text { as of } 1 / 1 / 98\end{array}$ & $\begin{array}{l}\text { Rem. Reserves } \\
\text { as of } 1 / 1 / 98\end{array}$ & $\begin{array}{l}\text { Est. Total Recovery } \\
\text { as of } 1 / 1 / 98\end{array}$ & $\begin{array}{l}\text { Res./Prod. } \\
\text { (years) }\end{array}$ \\
\hline 241 Means & Texas-Dist. 8 & 1934 & 4.213 & 275.6 & 25.3 & 300.9 & 6.0 \\
\hline 242 Midland Farms & Texas-Dist. 8 & 1944 & 1.488 & 261.0 & 7.6 & 268.6 & 5.1 \\
\hline 243 Penwell & Texas-Dist. 8 & 1926 & 1.585 & 122.4 & 7.8 & 130.2 & 4.9 \\
\hline 244 Sand Hills & Texas-Dist. 8 & 1931 & 2.210 & 268.0 & 13.0 & 281.0 & 5.9 \\
\hline 245 Shafter Lake & Texas-Dist. 8 & 1947 & 1.415 & 106.2 & 4.9 & 111.1 & 3.5 \\
\hline 246 Spraberry Trend & Texas-Dist. 8 & 1951 & 23.414 & 816.0 & 95.9 & 911.9 & 4.1 \\
\hline $247 \mathrm{TXL}$ & Texas-Dist. 8 & 1944 & 2.295 & 276.9 & 11.2 & 288.1 & 4.9 \\
\hline 248 Waddell & Texas-Dist. 8 & 1927 & 1.055 & 106.8 & 3.6 & 110.4 & 3.4 \\
\hline 249 Ward S. & Texas-Dist. 8 & 1938 & 0.356 & 109.1 & 1.5 & 110.6 & 4.2 \\
\hline 250 Ward Estes N. & Texas-Dist. 8 & 1929 & 2.405 & 391.1 & 23.5 & 414.6 & 9.8 \\
\hline 251 Westbrook & Texas-Dist. 8 & 1920 & 1.278 & 103.0 & 6.6 & 109.6 & 5.2 \\
\hline 252 Yates & Texas-Dist. 8 & 1926 & 20.310 & 1356.1 & 598.4 & 1954.5 & 29.5 \\
\hline 253 Adair & Texas-Dist. 8A & 1947 & 0.822 & 115.7 & 2.4 & 118.1 & 2.9 \\
\hline 254 Anton-Irish & Texas-Dist. 8A & 1944 & 1.527 & 196.4 & 10.1 & 206.5 & 6.6 \\
\hline 255 Cedar Lake & Texas-Dist. 8A & 1939 & 2.695 & 96.4 & 15.3 & 111.7 & 5.7 \\
\hline 256 Cogdell & Texas-Dist. 8A & 1949 & 0.491 & 299.7 & 3.7 & 303.4 & 7.5 \\
\hline 257 Diamond M & Texas-Dist. 8A & 1948 & 0.982 & 251.7 & 6.0 & 257.7 & 6.1 \\
\hline 258 Garza & Texas-Dist. 8A & 1926 & 2.075 & 127.2 & 9.1 & 136.3 & 4.4 \\
\hline 259 Jo-Mill & Texas-Dist. 8A & 1953 & 2.395 & 147.7 & 11.4 & 159.1 & 4.8 \\
\hline 260 Kelly-Snyder & Texas-Dist. 8A & 1948 & 3.145 & 1295.5 & 54.5 & 1350.0 & 17.3 \\
\hline 261 Levelland & Texas-Dist. 8A & 1938 & 11.080 & 615.7 & 48.6 & 664.3 & 4.4 \\
\hline 262 Prentice & Texas-Dist. 8A & 1951 & 3.105 & 198.6 & 24.5 & 223.1 & 7.9 \\
\hline 263 Robertson N. & Texas-Dist. 8A & 1956 & 5.102 & 113.4 & 28.9 & 142.3 & 5.7 \\
\hline 264 Salt Creek & Texas-Dist. 8A & 1950 & 9.191 & 331.5 & 46.4 & 377.9 & 5.0 \\
\hline 265 Seminole & Texas-Dist. 8A & 1936 & 11.950 & 656.3 & 57.5 & 713.8 & 4.8 \\
\hline 266 Sharon Ridge & Texas-Dist. 8A & 1923 & 1.502 & 100.6 & 10.6 & 111.2 & 7.1 \\
\hline 267 Slaughter & Texas-Dist. 8A & 1936 & 15.503 & 1177.9 & 77.9 & 1255.8 & 5.0 \\
\hline 268 Wasson & Texas-Dist. 8A & 1936 & 27.410 & 1964.1 & 122.5 & 2086.6 & 4.5 \\
\hline 269 Welch & Texas-Dist. 8A & 1942 & 2.351 & 167.7 & 20.1 & 187.8 & 8.5 \\
\hline 270 Archer Cnty Reg. & Texas-Dist. 9 & 1911 & 0.942 & 189.8 & 5.4 & 195.2 & 5.7 \\
\hline 271 Cooke Cnty Reg. & Texas-Dist. 9 & 1926 & 0.687 & 107.6 & 3.2 & 110.8 & 4.7 \\
\hline 272 K-M-A & Texas-Dist. 9 & 1931 & 0.833 & 147.6 & 2.6 & 150.2 & 3.1 \\
\hline 273 Walnut Bend & Texas-Dist. 9 & 1938 & 0.369 & 127.1 & 1.9 & 129.0 & 5.1 \\
\hline 274 Wichita Cnty Reg. & Texas-Dist. 9 & 1910 & 1.855 & 354.0 & 9.6 & 363.6 & 5.2 \\
\hline 275 Wilbarger Cnty Reg. & Texas-Dist. 9 & 1915 & 0.411 & 150.4 & 2.3 & 152.7 & 5.6 \\
\hline 276 Young Cnty Reg. & Texas-Dist. 9 & 1917 & 0.870 & 107.4 & 2.4 & 109.8 & 2.8 \\
\hline 277 Panhandle & Texas-Dist. 10 & 1921 & 5.125 & 1475.8 & 31.1 & 1506.9 & 6.1 \\
\hline 278 Altamont & Utah & 1955 & 1.613 & 112.2 & 15.9 & 128.1 & 9.9 \\
\hline 279 Bluebell & Utah & 1949 & 2.772 & 140.9 & 15.9 & 156.8 & 5.7 \\
\hline 280 Greater Aneth & Utah & 1956 & 5.874 & 405.0 & 25.0 & 430.0 & 4.3 \\
\hline 281 Byron & Wyoming & 1918 & 0.627 & 127.4 & 2.4 & 129.8 & 3.8 \\
\hline 282 Elk Basin & Wyoming & 1915 & 1.805 & 452.1 & 12.0 & 464.1 & 6.6 \\
\hline 283 Frannie & Wyoming & 1928 & 0.285 & 120.3 & 1.3 & 121.6 & 4.6 \\
\hline 284 Garland & Wyoming & 1906 & 2.022 & 182.9 & 7.7 & 190.6 & 3.8 \\
\hline 285 Grass Creek & Wyoming & 1914 & 1.450 & 201.4 & 5.6 & 207.0 & 3.9 \\
\hline 286 Hamilton Dome & Wyoming & 1918 & 1.778 & 249.5 & 5.8 & 255.3 & 3.3 \\
\hline 287 Hartzog Draw & Wyoming & 1976 & 2.111 & 96.5 & 12.1 & 108.6 & 5.7 \\
\hline 288 Lance Creek & Wyoming & 1918 & 0.125 & 109.1 & 0.9 & 110.0 & 7.2 \\
\hline 289 Little Buffalo Basin & Wyoming & 1914 & 0.797 & 131.3 & 3.0 & 134.3 & 3.8 \\
\hline 290 Lost Soldier & Wyoming & 1916 & 2.457 & 237.2 & 19.1 & 256.3 & 7.8 \\
\hline 291 Oregon Basin & Wyoming & 1912 & 4.431 & 441.8 & 19.6 & 461.4 & 4.4 \\
\hline 292 Salt Creek & Wyoming & 1889 & 2.159 & 658.6 & 17.5 & 676.1 & 8.1 \\
\hline \multirow[t]{2}{*}{293 Wertz } & Wyoming & 1921 & 0.509 & 113.4 & 8.7 & 122.1 & 17.1 \\
\hline & SUMS & & 1330.916 & 101758.7 & 11303.9 & 113062.6 & $\begin{array}{r}8.3 \\
\text { (ave) }\end{array}$ \\
\hline
\end{tabular}




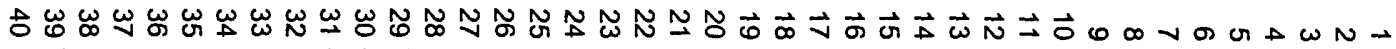
罾

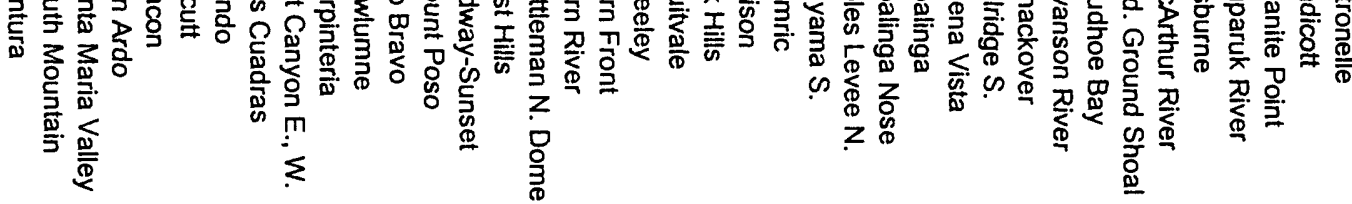

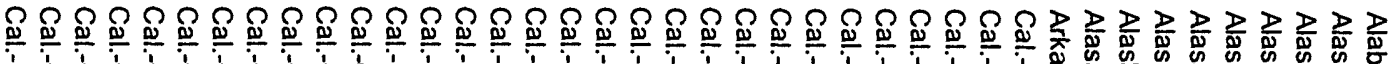

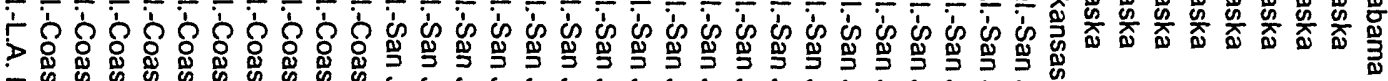

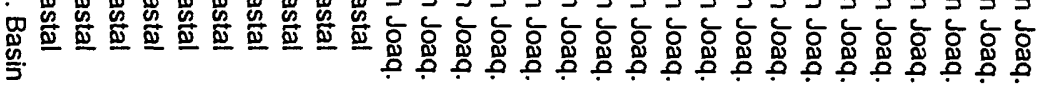

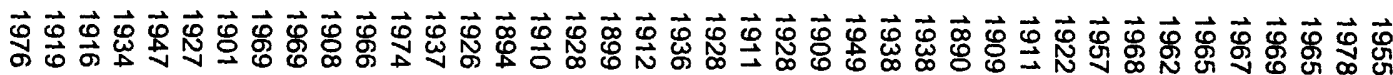

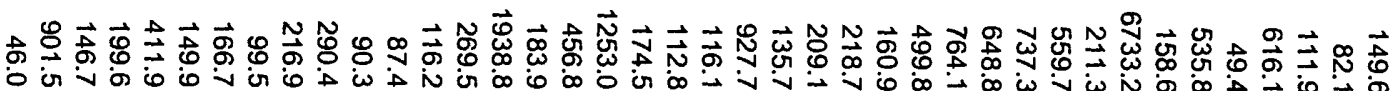

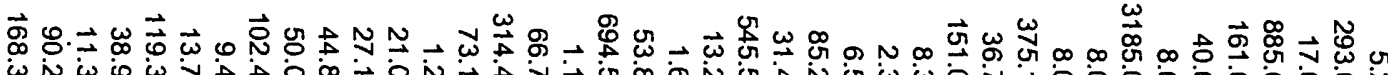

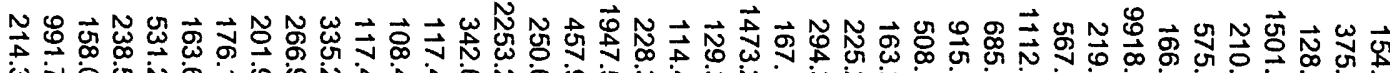

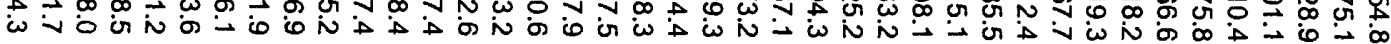

w

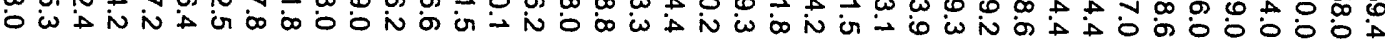

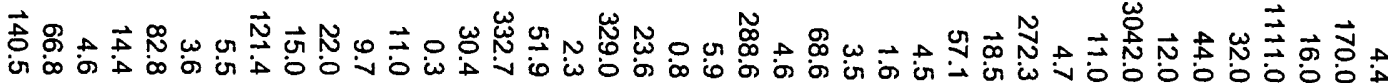

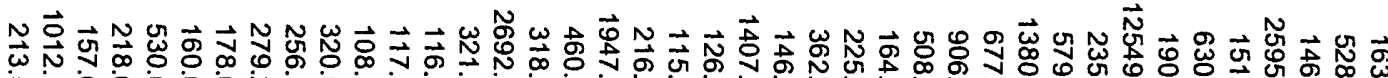

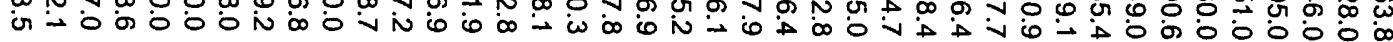

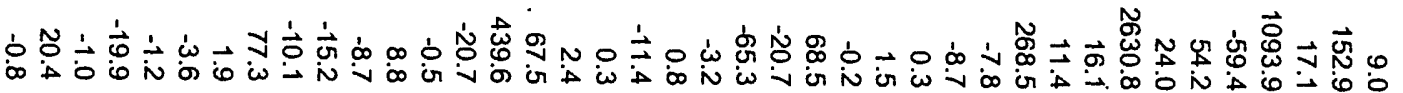




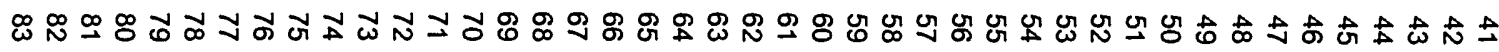

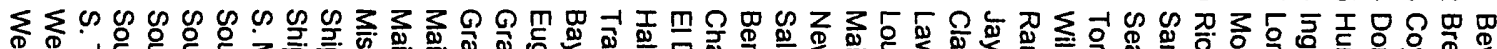

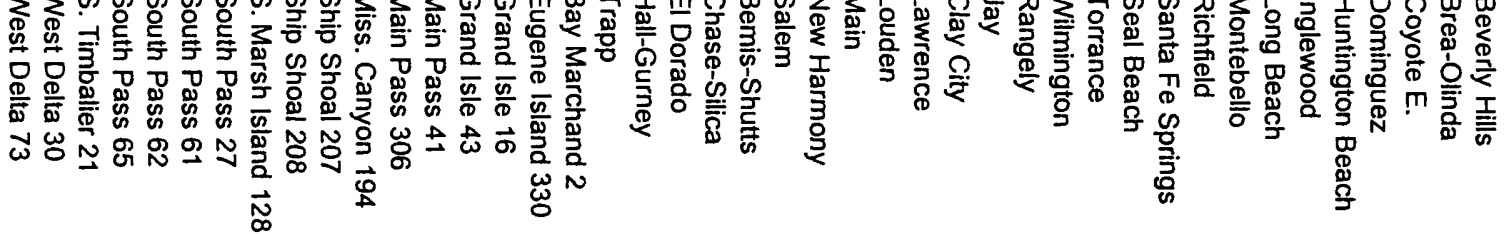

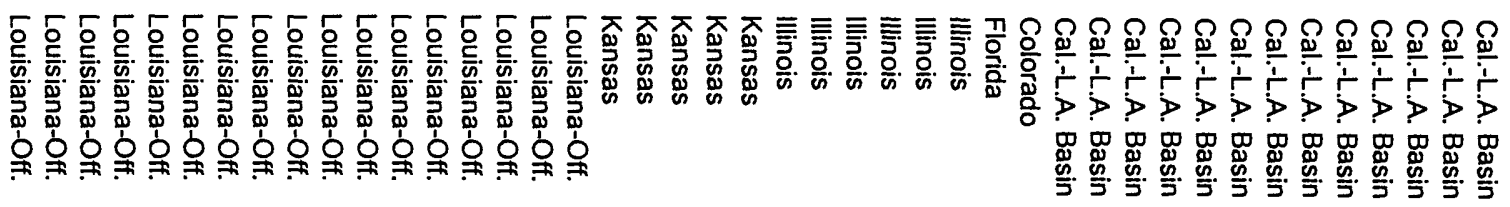

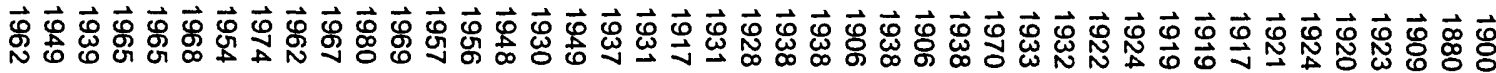

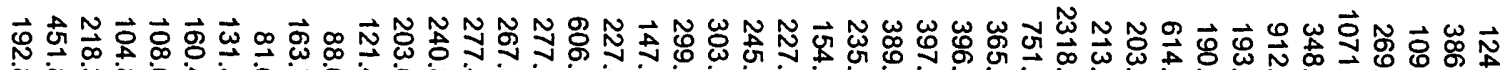
o i

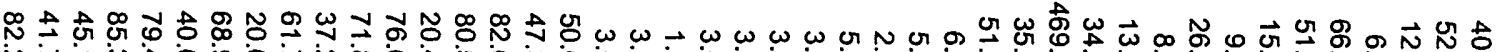

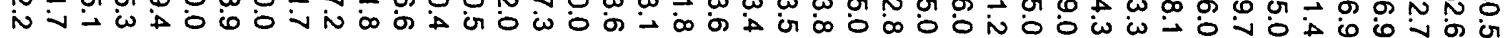

N

它

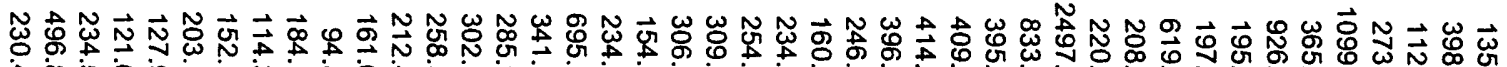

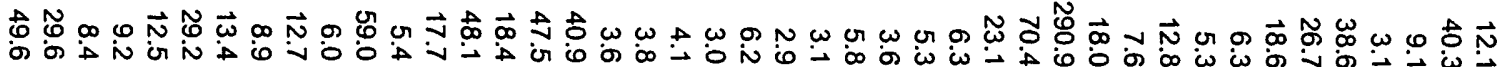

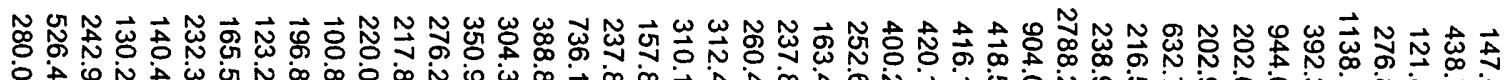

잉을 


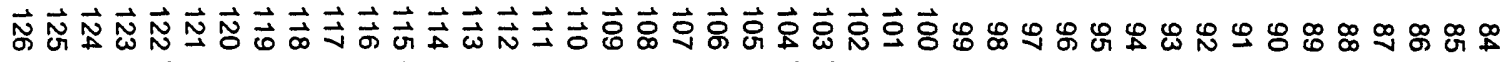

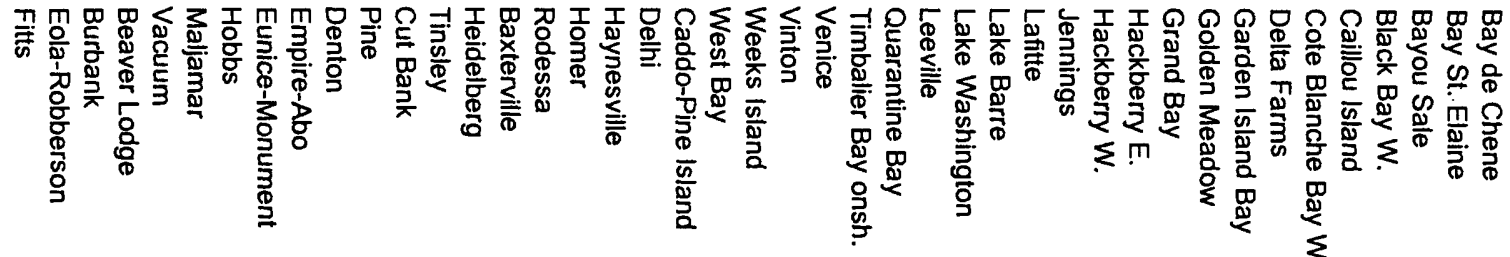

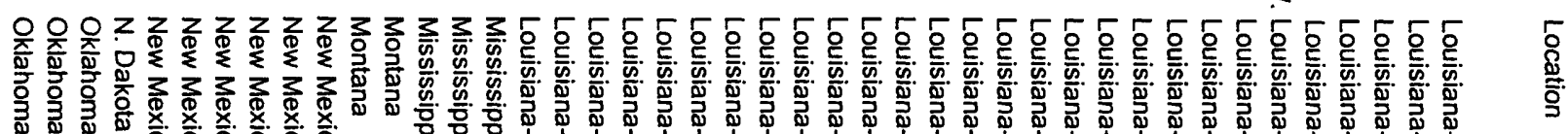

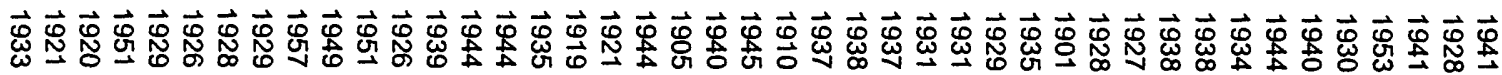

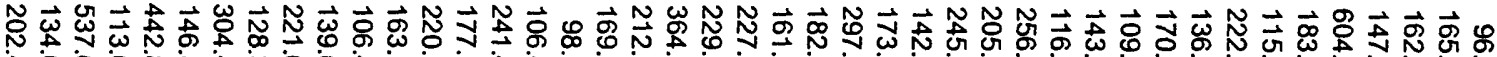
$\perp$ o

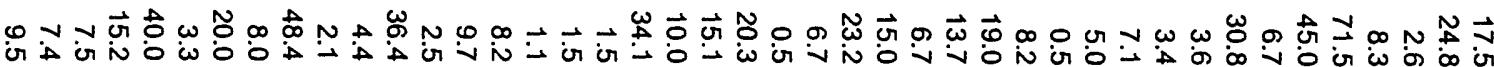

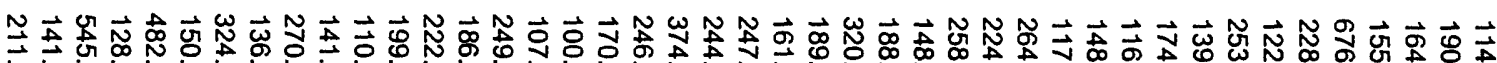

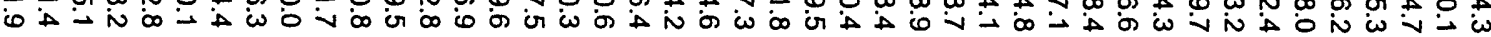

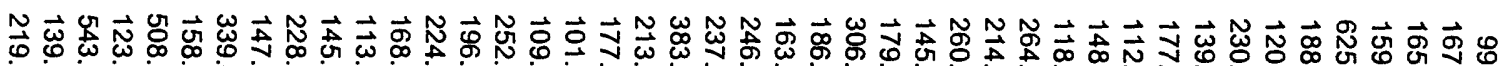

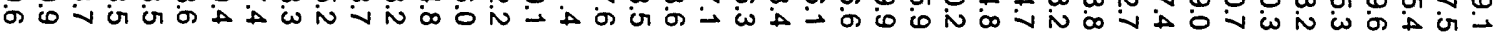

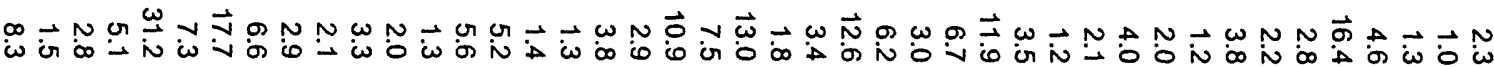

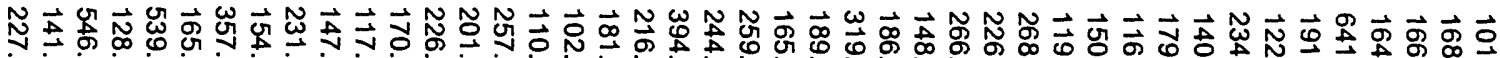

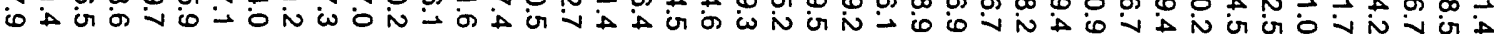

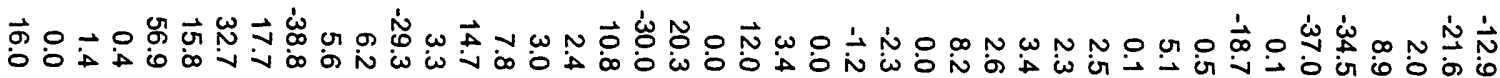




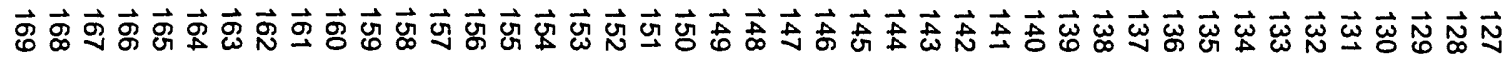

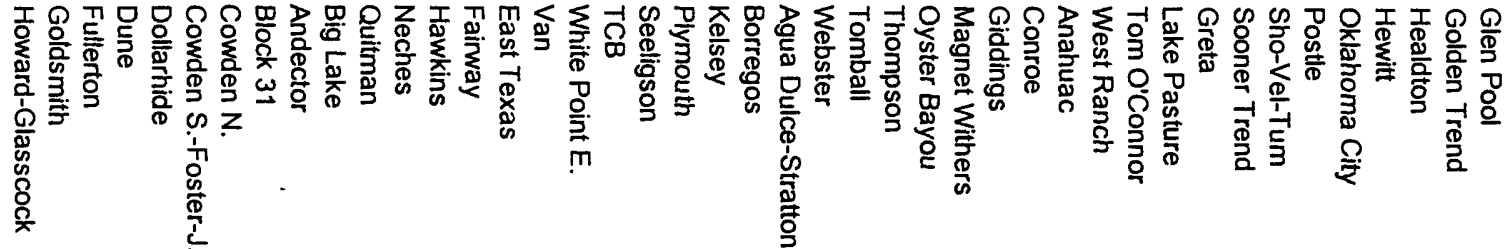

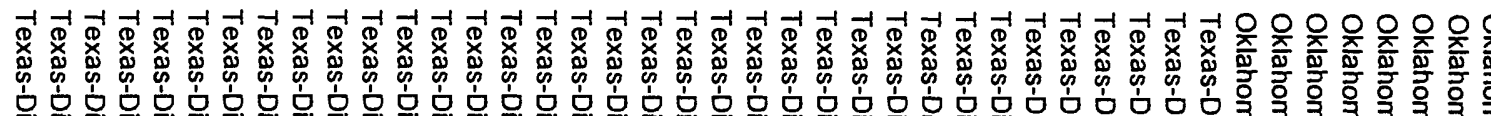

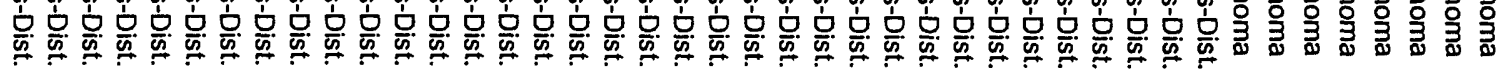

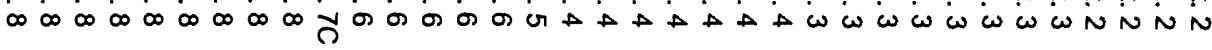

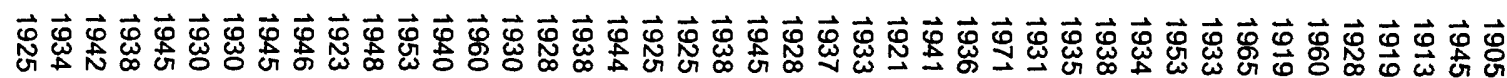

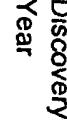

๓

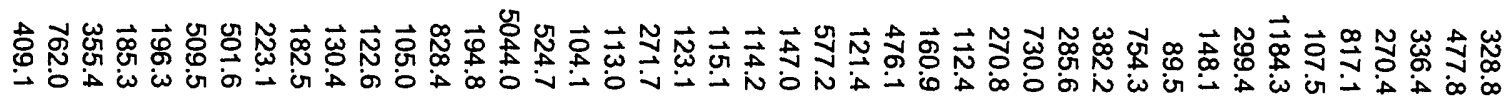

욕

富

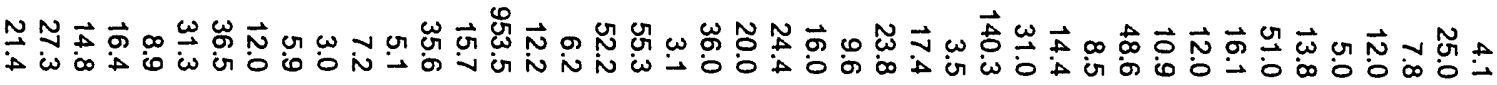

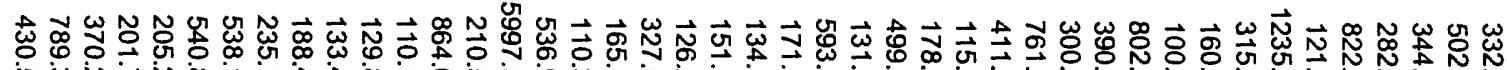

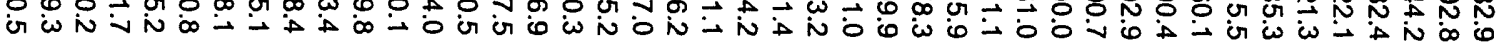

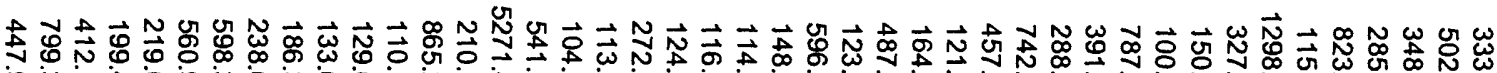

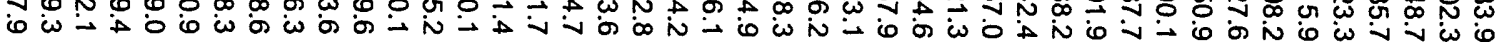

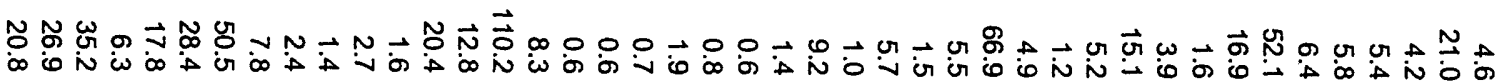

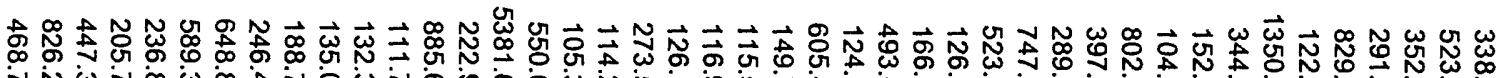

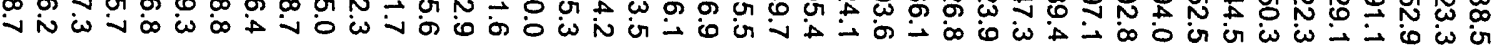

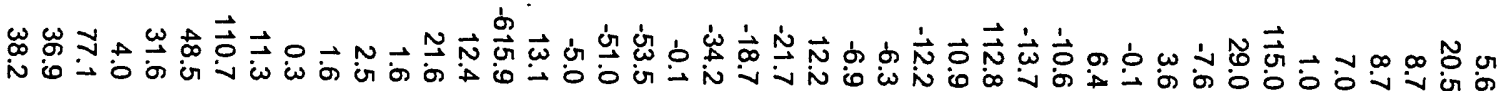




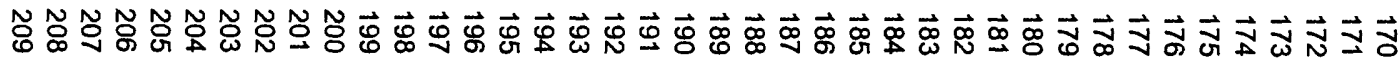

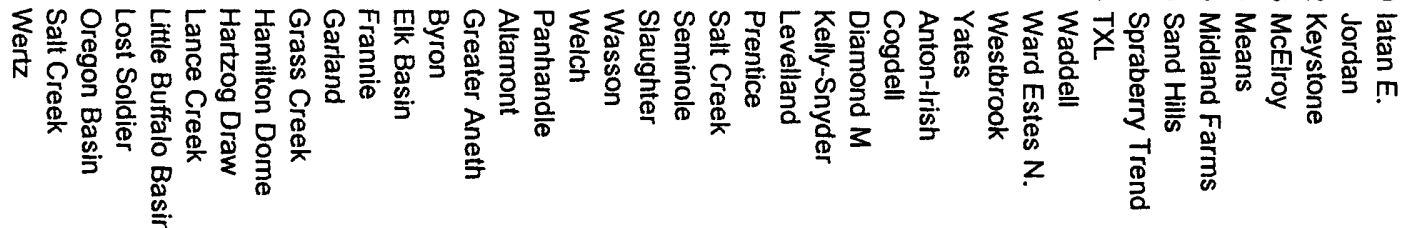

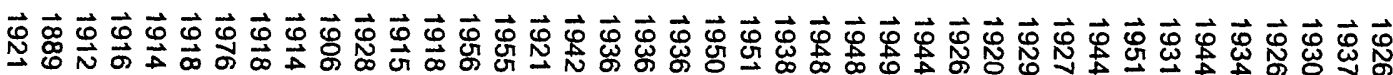

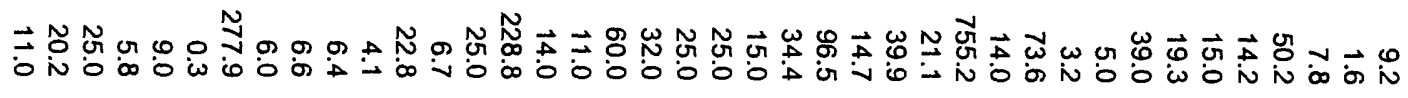

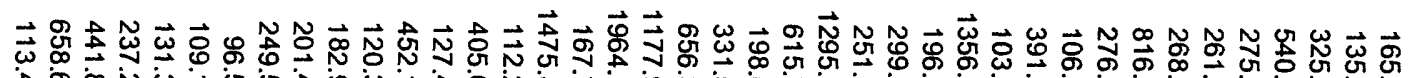

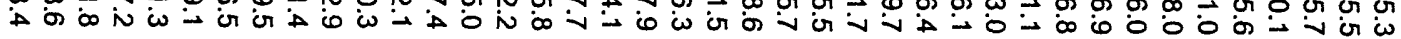

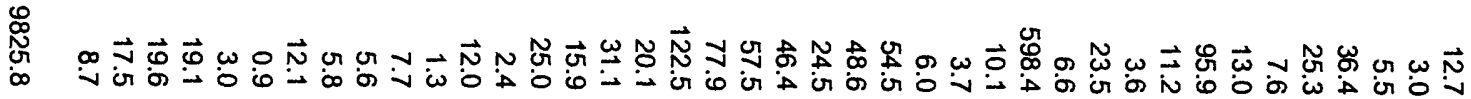

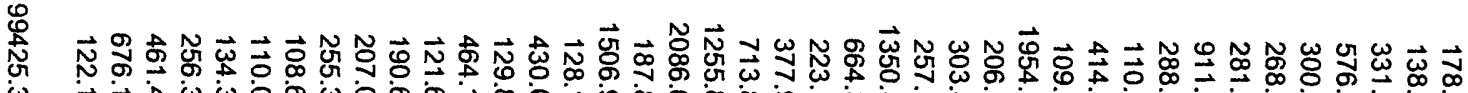

产 
Table 3. Giant U.S. oil fields of Table 1 having significant remaining potential (those that account for $80 \%$ of annual production or $80 \%$ of remaining reserves). [Data are sorted by rank with respect to annual production.

Rank with respect to remaining reserves is also shown. Production and reserves are in mmbo.]

ID No. Field

Table 1

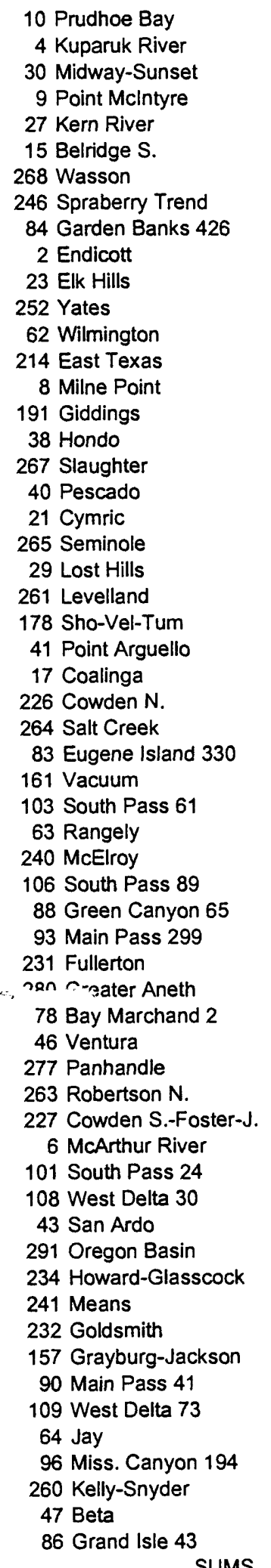

Rank- Rank- Production Rem. Reserves

production rem. reserves in 1997 as of $1 / 1 / 98$

\begin{tabular}{|c|c|c|c|}
\hline 1 & 1 & 251.000 & 3042.0 \\
\hline 2 & 2 & 96.000 & 1111.0 \\
\hline 3 & 4 & 60.692 & 332.7 \\
\hline 4 & 9 & 59.000 & 241.0 \\
\hline 5 & 5 & 48.746 & 329.0 \\
\hline 6 & 8 & 42.848 & 272.3 \\
\hline 7 & 14 & 27.410 & 122.5 \\
\hline 8 & 17 & 23.414 & 95.9 \\
\hline 9 & 18 & 22.706 & 93.4 \\
\hline 10 & 11 & 21.000 & 170.0 \\
\hline 11 & 7 & 20.529 & 288.6 \\
\hline 12 & 3 & 20.310 & 598.4 \\
\hline 13 & 6 & 19.779 & 290.9 \\
\hline 14 & 16 & 19.213 & 110.2 \\
\hline 15 & 10 & 19.000 & 223.0 \\
\hline 16 & 23 & 16.590 & 66.9 \\
\hline 17 & 15 & 16.491 & 121.4 \\
\hline 18 & 20 & 15.503 & 77.9 \\
\hline 19 & 31 & 15.161 & 51.7 \\
\hline 20 & 22 & 13.847 & 68.6 \\
\hline 21 & 26 & 11.950 & 57.5 \\
\hline 22 & 30 & 11.520 & 51.9 \\
\hline 23 & 34 & 11.080 & 48.6 \\
\hline 24 & 29 & 10.800 & 52.1 \\
\hline 25 & 12 & 10.563 & 160.7 \\
\hline 26 & 27 & 9.732 & 57.1 \\
\hline 27 & 32 & 9.595 & 50.5 \\
\hline 28 & 38 & 9.191 & 46.4 \\
\hline 29 & 36 & 8.655 & 47.5 \\
\hline 30 & 48 & 8.015 & 31.2 \\
\hline 31 & 53 & 7.914 & 29.2 \\
\hline 32 & 21 & 7.529 & 70.4 \\
\hline 33 & 45 & 7.455 & 36.4 \\
\hline 34 & 41 & 7.290 & 41.6 \\
\hline 35 & 37 & 7.133 & 46.5 \\
\hline 36 & 39 & 6.903 & 45.1 \\
\hline 37 & 46 & 6.655 & 35.2 \\
\hline 38 & 62 & 5.874 & 25.0 \\
\hline 39 & 42 & 5.311 & 40.9 \\
\hline 40 & 24 & 5.191 & 66.8 \\
\hline 41 & 49 & 5.125 & 31.1 \\
\hline 42 & 54 & 5.102 & 28.9 \\
\hline 43 & 56 & 5.015 & 28.4 \\
\hline 44 & 40 & 5.000 & 44.0 \\
\hline 45 & 57 & 4.850 & 27.9 \\
\hline 46 & 52 & 4.779 & 29.6 \\
\hline 47 & 19 & 4.629 & 82.8 \\
\hline 48 & 74 & 4.431 & 19.6 \\
\hline 49 & 71 & 4.215 & 20.8 \\
\hline 50 & 61 & 4.213 & 25.3 \\
\hline 51 & 59 & 4.198 & 26.9 \\
\hline 52 & 79 & 4.059 & 18.3 \\
\hline 53 & 82 & 3.841 & 17.7 \\
\hline 54 & 33 & 3.803 & 49.6 \\
\hline 55 & 67 & 3.777 & 23.1 \\
\hline 59 & 25 & 3.213 & 59.0 \\
\hline 61 & 28 & 3.145 & 54.5 \\
\hline 64 & 13 & 3.009 & 140.5 \\
\hline \multirow[t]{2}{*}{68} & 35 & 2.884 & 48.1 \\
\hline & & 1076.883 & 9524.1 \\
\hline
\end{tabular}

